RadioGraphics 2009; e38 • Published online 10.1148/rg.e38 - Content Codes: GI MR

\title{
The Hypointense Liver Lesion on T2-Weighted MR Images and What It Means ${ }^{1}$
}

\author{
Luís Curvo-Semedo, MD, Jorge B. Brito, MD, Miguel F. Seco, MD, João F. Costa, MD, Cristina
}

B. Marques, MD, Filipe Caseiro-Alves, MD, PhD

${ }^{1}$ From the Department of Radiology, Coimbra University Hospital, Praceta Mota Pinto/Av. Bissaya Barreto, 3000-075 Coimbra, Portugal. Received March 23, 2009; revision requested June 22 and received July 13; accepted August 4. Authors have no financial relationships to disclose. Presented as an education exhibit at the 2008 RSNA annual meeting. Address correspondence to L.C.S. (e-mail: curvosemedo@gmail.com).

TEACHING POINTS

See last page

\section{Abbreviations}

$\mathrm{FNH}=$ focal nodular hyperplasia, $\mathrm{FSE}=$ fast spin echo, $\mathrm{HCC}=$ hepatocellular carcinoma, $\mathrm{SI}=$ signal intensity.

\section{ABSTRACT}

The vast majority of focal liver lesions are hyperintense on T2-weighted magnetic resonance (MR) images. Rarely, however, hepatic nodules may appear totally or partially hypointense on those images. Causes for this uncommon appearance include deposition of iron, calcium, or copper and are related to the presence of blood degradation products, macromolecules, coagulative necrosis, and other conditions. Although rare, low signal intensity relative to surrounding liver on T2-weighted images may be seen in a wide spectrum of lesions. Examples include cases of focal nodular hyperplasia, hepatocellular adenoma, hepatocellular carcinoma, metastases, leyomioma, siderotic or dysplastic nodules, nodules in Wilson disease, granuloma, and hydatid cyst. On fat-suppressed T2-weighted images, nodules with a lipomatous component, such as lipoma, angiomyolipoma, hepatocellular adenoma, and hepatocellular carcinoma may also appear partially or totally hypointense. The conjunction of other MR imaging findings and their integration in the clinical setting may allow a correct diagnosis in a considerable proportion of cases. The cause for T2-weighted hypointensity may not be, however, always recognized, and only pathologic correlation may provide the answer. The aims of this work are to discuss the causes and mechanisms of hypointensity of liver lesions on T2-weighted images and proposing an algorithm for classification that may be useful as a quick reminder for the interested reader.

\section{INTRODUCTION}

Magnetic resonance (MR) imaging of liver lesions is of pivotal importance in clinical practice. In abdominal MR imaging, T1- and T2-weighted sequences remain a basic requirement for lesion characterization. MR signal intensity (SI) of focal liver lesions is affected by numerous pathologic factors. Histologic features strongly affect T1 and T2 relaxation times and MR imaging is unique in its ability to explore intracellular content and recognize the presence of substances that may govern signal behavior. Owing to their high cellular water content, the majority of focal liver lesions appear hyperintense on T2-weighted images. In contrast to this most frequently seen MR imaging pattern, liver nodules may show total or partial hypointensity on T2-weighted images. The hypointense appearance can be classified as absolute or relative, depending on the native SI of adjacent liver parenchyma and sequence parameters (Fig 1). Although relative hypointensity depends on the native SI of liver, which can be modified by several circumstances (eg, inflammation, diffuse iron deposition, etc), it may be more commonly encountered when 
lesions possess a fat component and fat-nulling imaging techniques are used. Radiologists should be aware of the various causes and mechanisms of hypointensity of focal liver lesions on T2weighted images and be able to recognize the spectrum of focal liver lesions presenting this imaging finding.

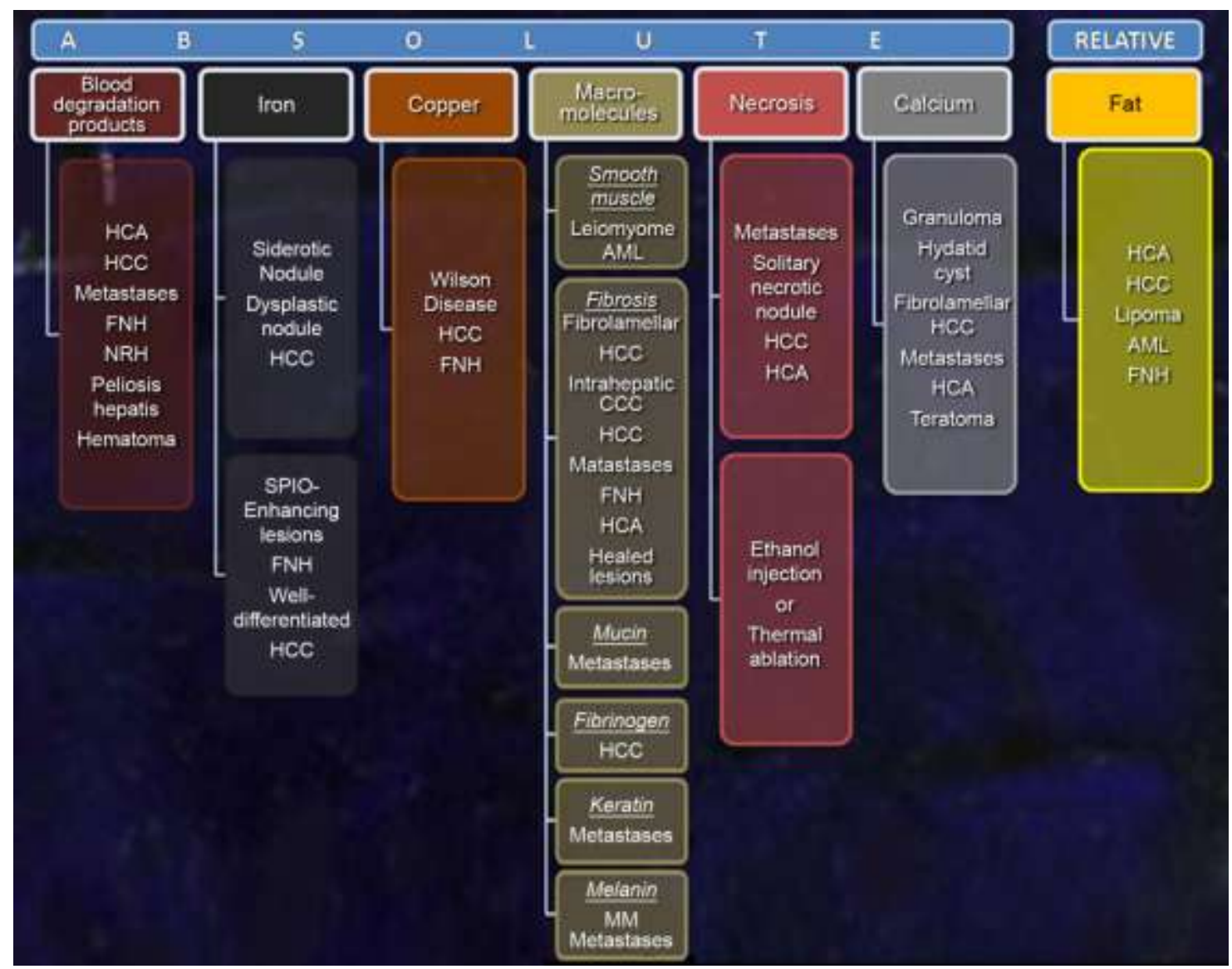

Figure 1. Causes of hypointensity on T2-weighted images. The hypointense appearance can be absolute or relative, depending on the native SI of adjacent liver parenchyma and the sequences used. Causes for absolute hypointensity are found in multiple lesions, whereas relative hypointensity depends on the native SI of the liver (which can be modified by circumstances such as inflammation, diffuse iron deposition, etc) and may be more commonly encountered when the lesions possess a fatty component and fat-nulling techniques are used. $A M L=$ angiomyolipoma, $C C C=$ cholangiocarcinoma, $H C A$ = hepatocellular adenoma, $N R H=$ nodular regenerative hyperplasia, $S P I O=$ superparamagnetic iron oxide.

\section{IMAGING FINDINGS}

Several causes for a hypointense appearance of focal liver lesions on T2-weighted images can be determined.

\section{Blood Degradation Products}

The MR imaging appearance of hemorrhage is related to the presence of different stages of blood breakdown products within the lesion (1). Acute hemorrhage shows an SI behavior that is consistent with intracellular deoxyhemoglobin: low on T1-weighted images and markedly low on T2weighted images. Subacute hemorrhage (within 3-5 days) indicates the presence of intracellular methemoglobin: high SI on T1-weighted images resulting from the paramagnetic effects of me- 
themoglobin and variable SI on T2-weighted images. The T2-weighted SI of methemoglobin depends on whether it is located in an intra- or extracellular environment; the former results in low SI (marked T2 shortening), while the latter causes high SI (less T2 shortening). In later stages, hemorrhage may have low SI on both T2- and T1-weighted sequences, since the intracellular forms of iron ferritin or hemosiderin provoke marked SI loss due to magnetic susceptibility effects (1).

Hepatocellular adenomas are prone to bleeding and consequently show SI heterogeneity resulting from the combination of hemorrhage and necrosis (2), but this occurs in a minority of cases. As discussed above, one may expect that old bleeding zones display low SI on T2-weighted images. The same mechanism applies to hepatocellular carcinoma (HCC), which may show internal bleeding (Fig 2), as well as to some metastatic lesions (Fig 3).

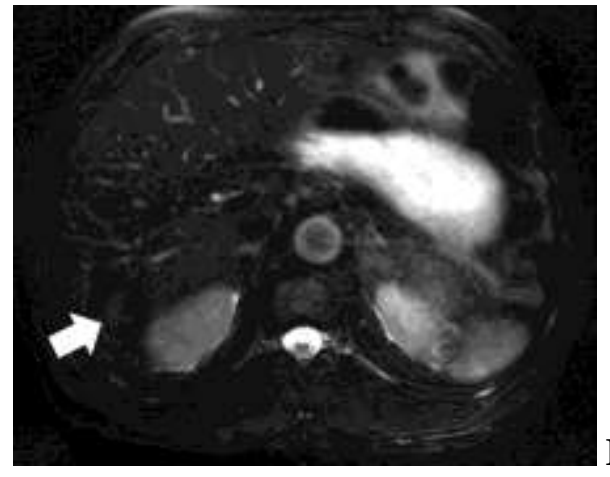

Fig 2a
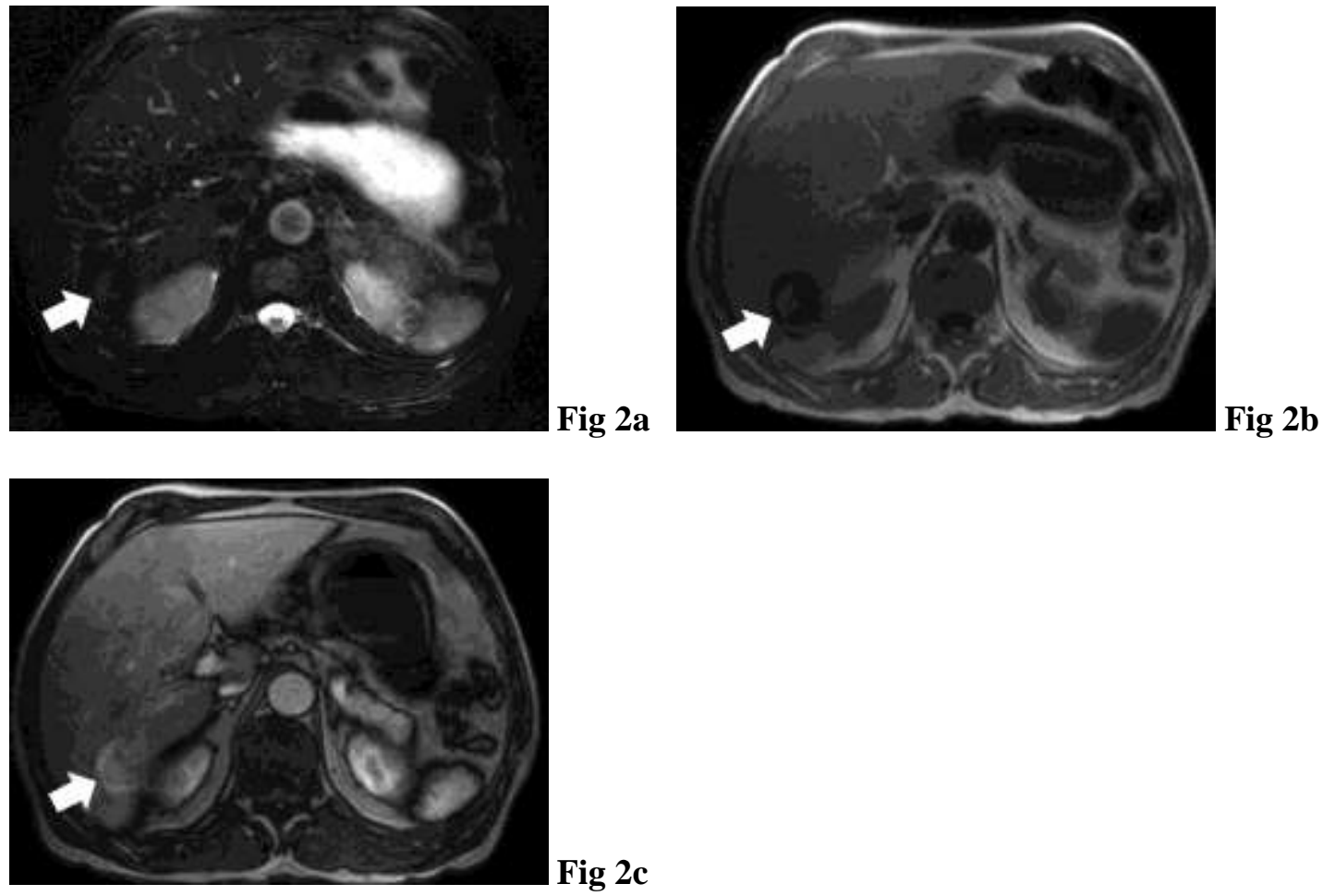

Figure 2. HCC with hemorrhagic foci in a 64-year-old man with hepatitis C cirrhosis. (a-c) Heterogeneous mass (arrow) is seen in right liver lobe, predominantly hypointense on (a) T2-weighted FSE image (repetition time, $1800 \mathrm{msec} / \mathrm{echo}$ time, $93 \mathrm{msec}$ ) and (b) in-phase T1-weighted image (100/2.4). (c) On the arterial-phase image of the dynamic study, the lesion shows marked heterogeneous enhancement, with a non-enhancing central area. The biopsy specimen yielded abundant hemosiderin deposits consistent with old hemorrhage. 

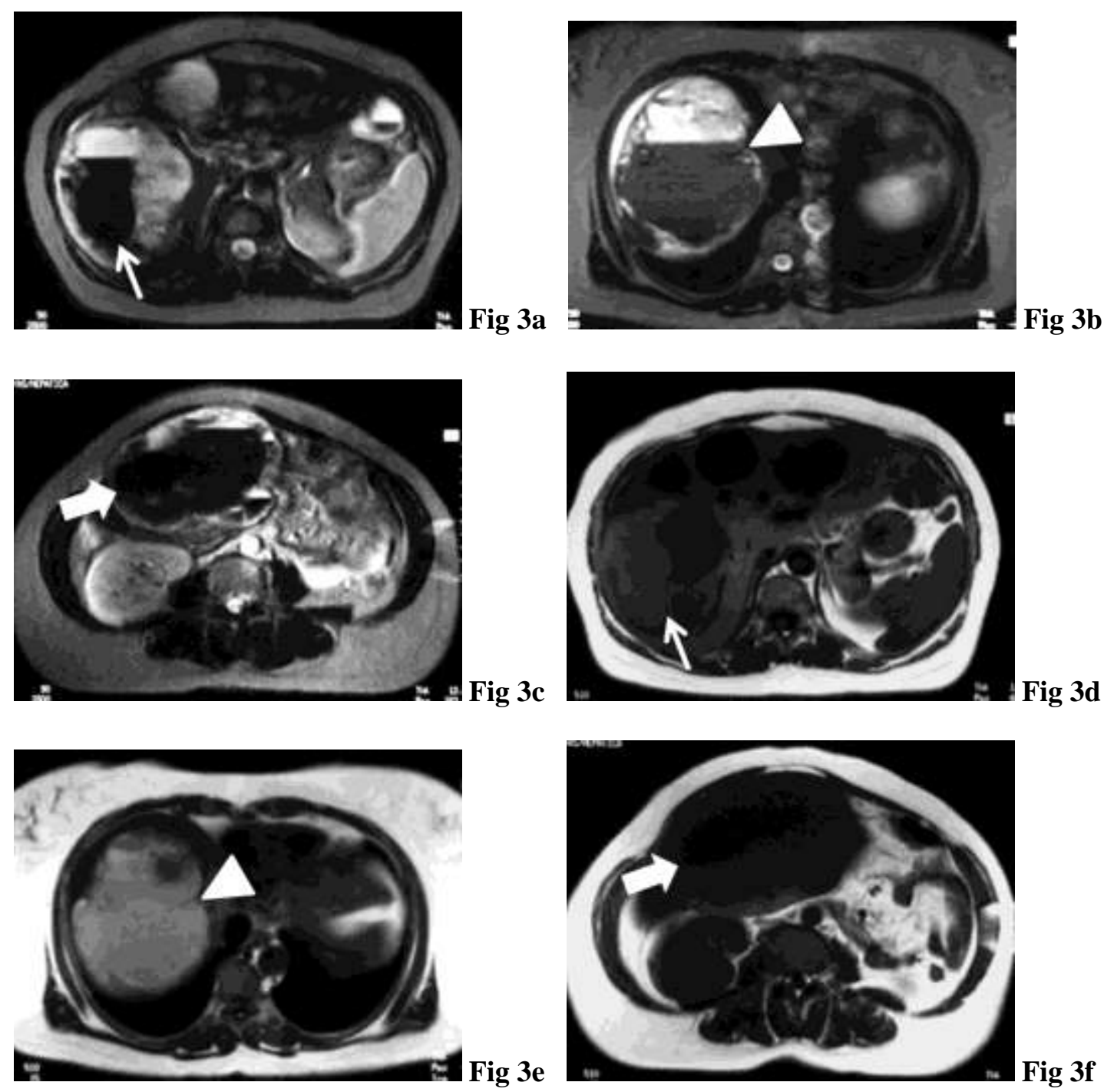

Figure 3. Hemorrhagic metastases in a 54-year-old woman with pancreatic neuroendocrine tumor. (a-f) Several heterogeneous liver masses are seen dispersed throughout the liver, showing several degrees of hypointensity on (a-c) T2-weighted images (2500/90), reflecting hemorrhages of different ages. (d-f) Note the variable SI on the corresponding T1-weighted (510/15) images. It is possible to appreciate acute bleeding, which is very dark on T2-weighted images (arrow, a) and discretely hypointense on T1weighted images (arrow, d). Metastases with subacute hemorrhage are seen with variable SI on T2weighted images (arrowhead, b) and high SI on T1-weighted images (arrowhead, e). It is also possible to recognize signs of chronic bleeding, which is hypointense with both sequences (arrow, $c, f$ ). These lesions represent cystic metastases, and in some it is possible to appreciate an internal fluid-fluid level. 
Despite being unusual, hypointensity of focal nodular hyperplasia $(\mathrm{FNH})$ on T2-weighted images can be found. Although the exact mechanism is not known, since no macroscopic acute bleeding is demonstrated, low SI may be the result of intratumoral hemolysis associated with rapid tumor growth (3) (Fig 4). Blood exerting its T2* effect on T2-weighted sequences is also seen in large hyperplastic nodules of Budd-Chiari syndrome caused by hemorrhage, infarction, or possibly venous congestion (Fig 5) (4,5). Hypointense areas on T2-weighted images can be found in peliosis hepatis when hemorrhage occurs in its large vascular spaces (Fig 6). As also discussed above, chronic hematomas may appear dark with T2-weighted sequences (Fig 7).

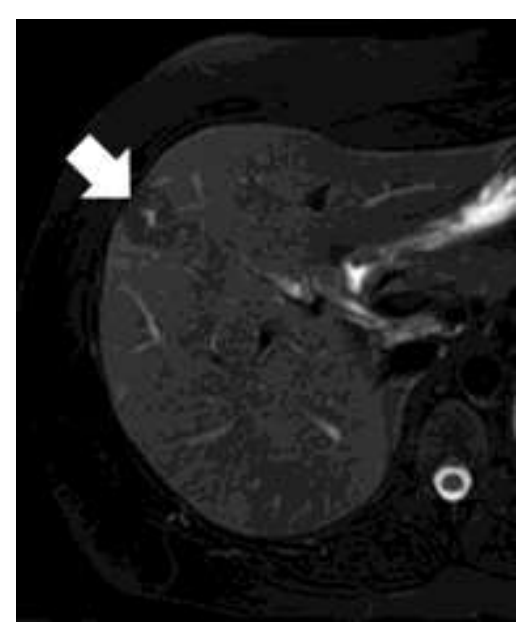

Fig 4a
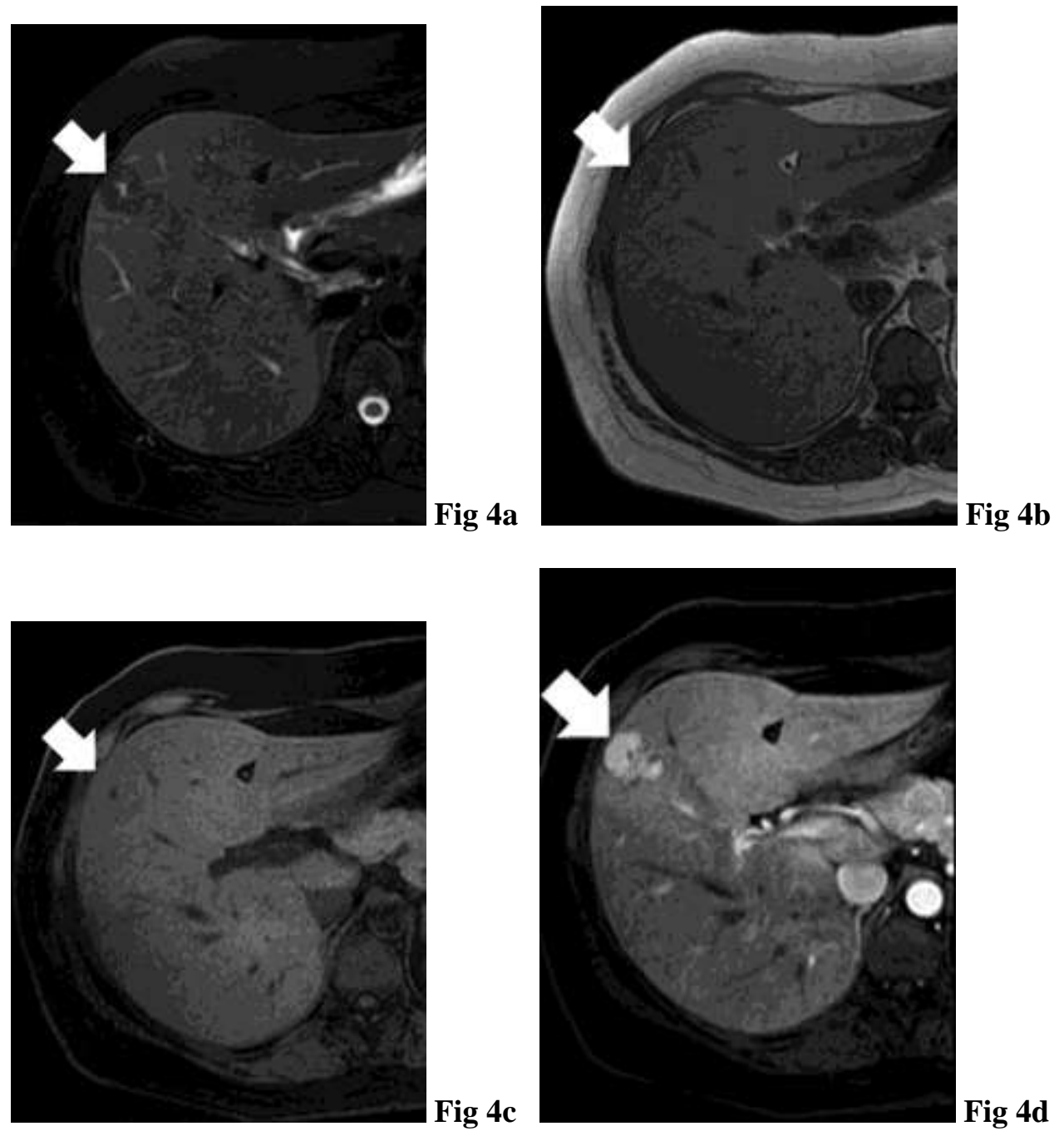

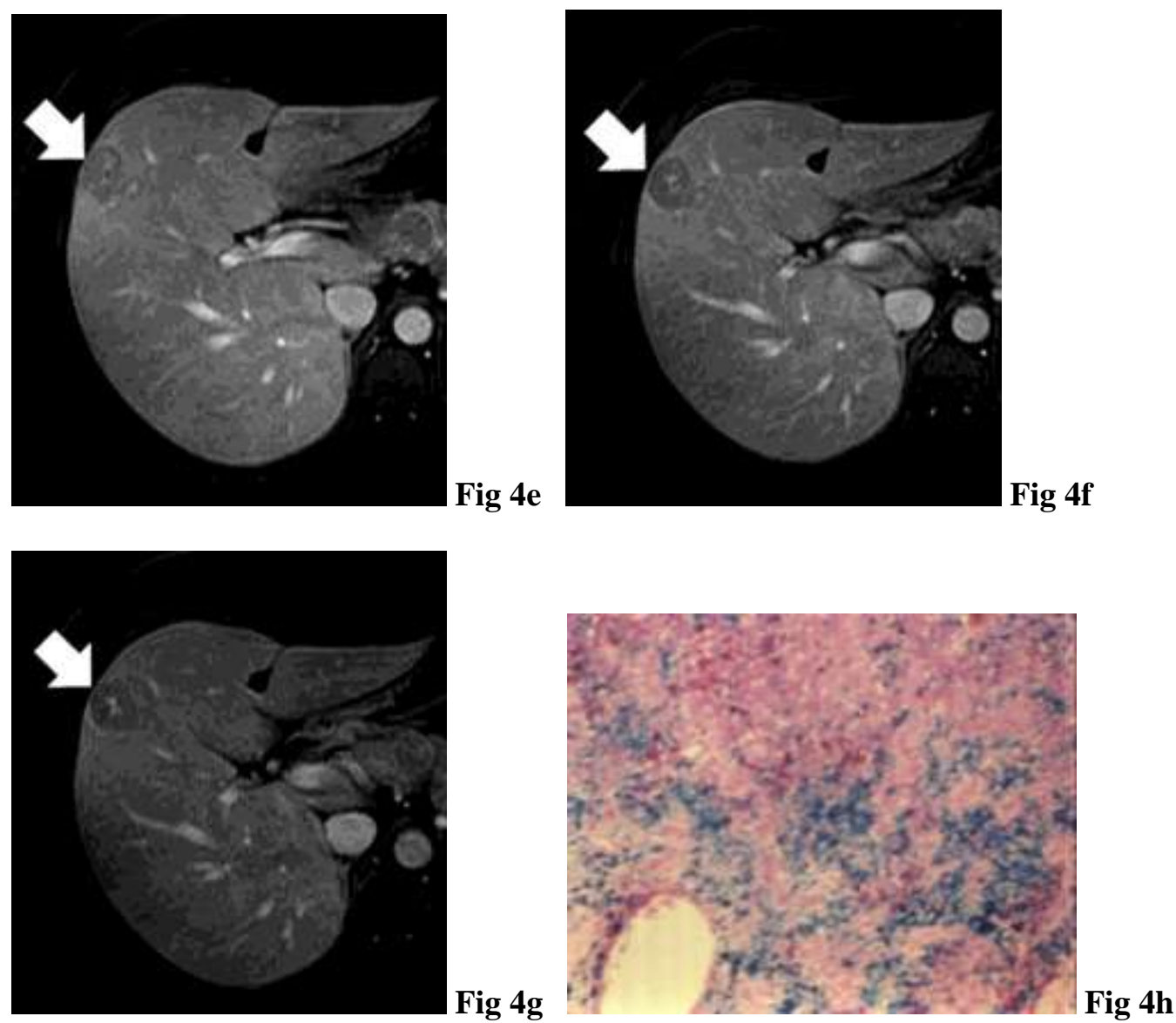

Figure 4. FNH in a 28-year-old woman. (a-g) Focal liver lesion (arrow) is hypointense on (a) T2-weighted FSE image (1800/93), with a hyperintense central scar; isointense to surrounding parenchyma on (b) T1weighted image (100/2.4) and (c) three-dimensional T1-weighted FSE image (3.64/1.44), with low SI in the central scar in c. (d-g) In images from the dynamic study, the lesion demonstrates a hypervascular behavior and late enhancement of the scar. (h) Pathologic study (original magnification, $\times 200$ ) revealed abundant iron deposits with Perl staining.
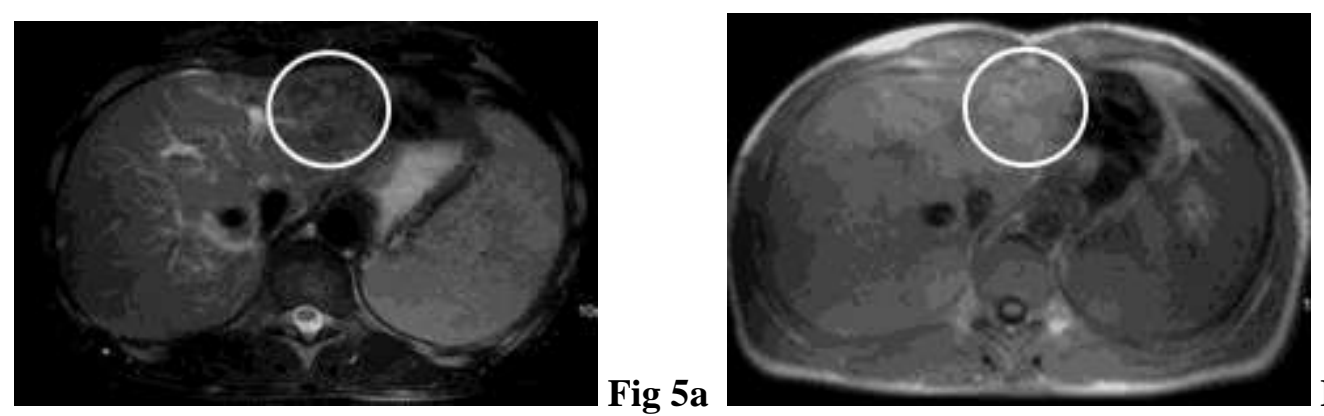

Fig $5 b$ 


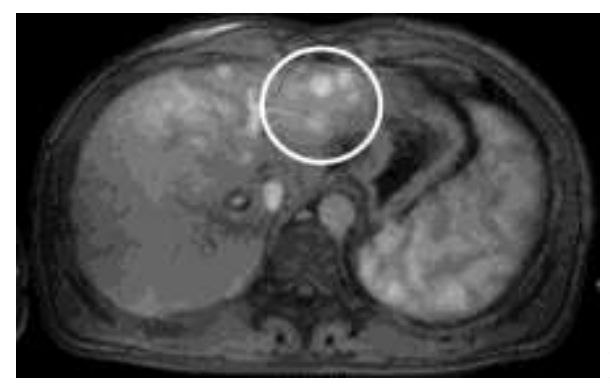

Fig $5 c$

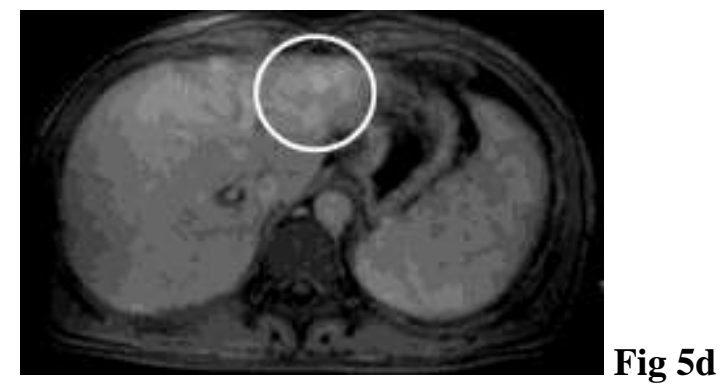

Figure 5. Hyperplastic nodules in a 34-year-old man with chronic Budd-Chiari disease secondary to polycythemia vera. (a-d) Several heterogeneous liver nodules (circles) can be recognized, some hypointense and others with a thick low-intensity peripheral rim on (a) T2-weighted FSE image (1800/93), possibly due to hemorrhage. (b) Note the "mirrored" SI pattern on the corresponding T1-weighted image (100/5.2). (c, d) On images from the dynamic study, these lesions show hypervascular features.

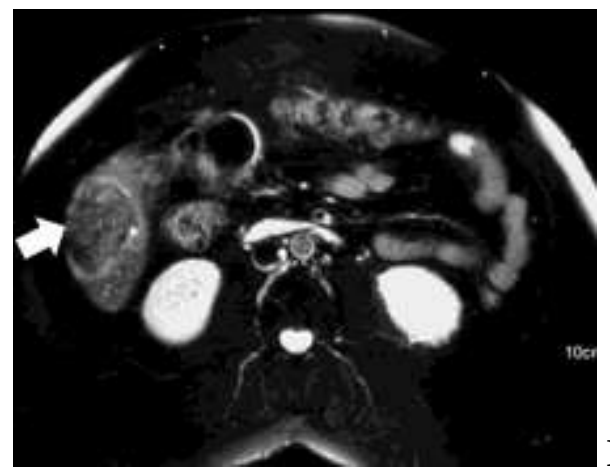

Fig 6a

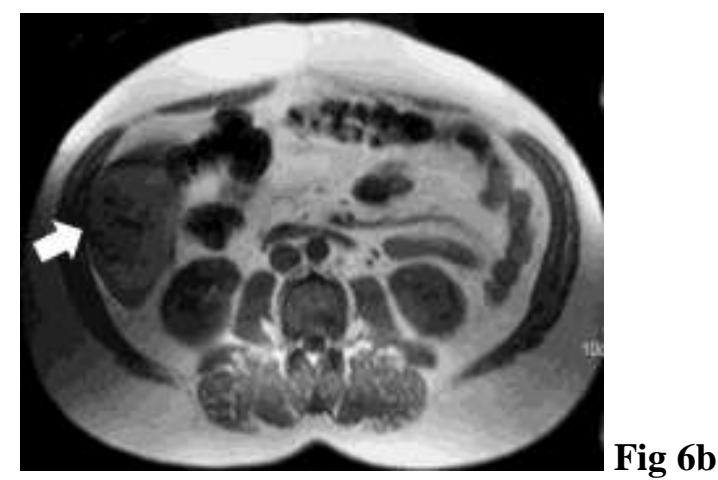

Figure 6. Peliosis hepatis in a 43-year-old woman. (a, b) In this focal form of peliosis hepatis (arrow), the lesion is heterogeneous, with areas of low SI on (a) T2-weighted FSE image (1800/93) and (b) T1weighted image (100/5.2). The biopsy specimen revealed the presence of hemosiderin within the hepatocyte cytoplasm, consistent with old hemorrhage.

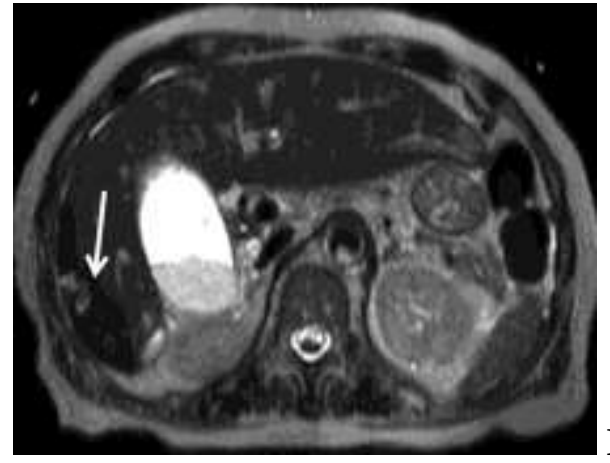

Fig 7a

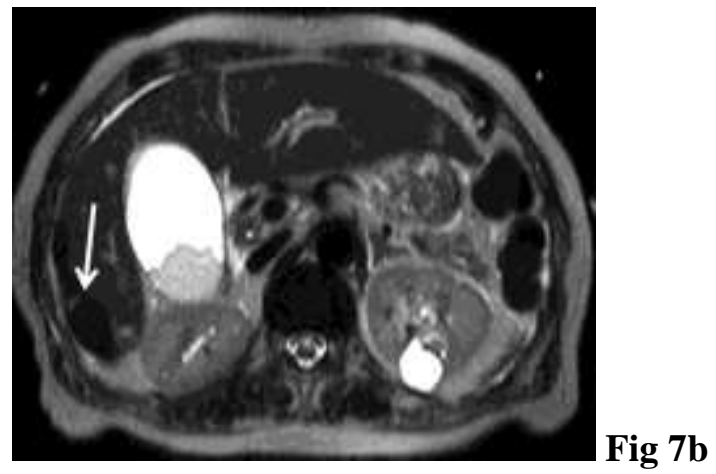

Figure 7. Subcapsular hematoma in a 55-year-old woman. (a, b) A predominantly dark, elongated collection (arrow) corresponding to a chronic subcapsular hematoma is seen on T2-weighted half-Fourier single-shot turbo spin-echo images obtained at different levels. 
Teaching Point

\section{Iron Deposition}

Iron in the liver is in the form $\mathrm{Fe}^{3+}$, which is paramagnetic and thus may produce hypointensity on T2-weighted images (6). Intracellular iron particles cause local field inhomogeneities that accelerate the $\mathrm{T} 2 *$ of tissues by rapid dephasing of the transverse magnetization. This effect can be appreciated with sequences that are sensitive to magnetic field inhomogeneities, such as T2weighted spin echo or, particularly, T2*-weighted gradient echo. T2-weighted FSE sequences include multiple refocusing $\left(180^{\circ}\right)$ pulses that compensate to some extent for the susceptibility effect and thus are less sensitive for iron detection. Conversely, the structure of gradient-echo sequences, devoid of refocusing radiofrequency pulses, makes them exquisitely sensitive to the presence of even small amounts of iron. By selecting a sufficiently long echo time and a small flip angle, the $\mathrm{T} 2 *$ effect can be maximized. It should be noted that using longer echo times causes artifactual enlargement of the iron-containing lesions, the so called "blooming effect" (7). Regenerative siderotic nodules in liver cirrhosis appear hypointense on T2-weighted images, owing to accumulation of intracellular iron (8,9) (Fig 8). Iron may also explain the hypointensity of dysplastic nodules on T2-weighted images when compared with adjacent liver parenchyma, but other causes should be considered, such as the presence of copper (10) (Fig 9). It is assumed that neoplastic tissues are unable to retain iron (11) despite their high content of ferritin (12). However, it has been shown that ferritin synthesis and secretion are stimulated by iron and, since ferritin acts as an intracellular iron store, cancer cells may occasionally take up iron (13) (Fig 10). The iron status of HCC cells correlates with intracellular levels of ferritin and may not be related to histologic grading of the tumor (14). After administration of reticuloendothelialsystem-targeted agents (superparamagnetic iron oxide), intracellular iron may be present in hepatocellular lesions containing Kupffer cells, such as FNH (Fig 11) (15); however, this is not specific, since hepatocellular adenoma or well-differentiated HCC may also show uptake of iron oxide particles (8). Liver lesions with negligible or abnormal reticuloendothelial cells remain mostly unchanged, while normal liver shows a homogeneous signal loss on T2-weighted images. This results in an improvement in the contrast-to-noise ratio for distinguishing liver and focal liver lesions (16).

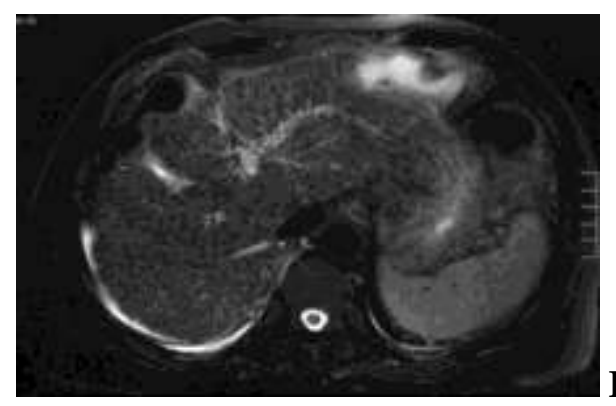

Fig 8a

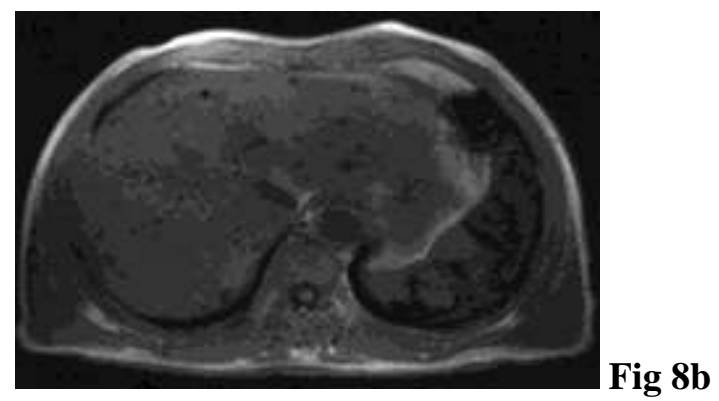

Figure 8. Regenerative siderotic nodules in a 53-year-old man with alcoholic cirrhosis. Tiny hypointense liver nodules dispersed throughout the liver parenchyma are seen on (a) T2-weighted FSE image (1800/93) and (b) T1-weighted image (100/5.2). These lesions have low SI on T2-weighted images because of their iron content. 

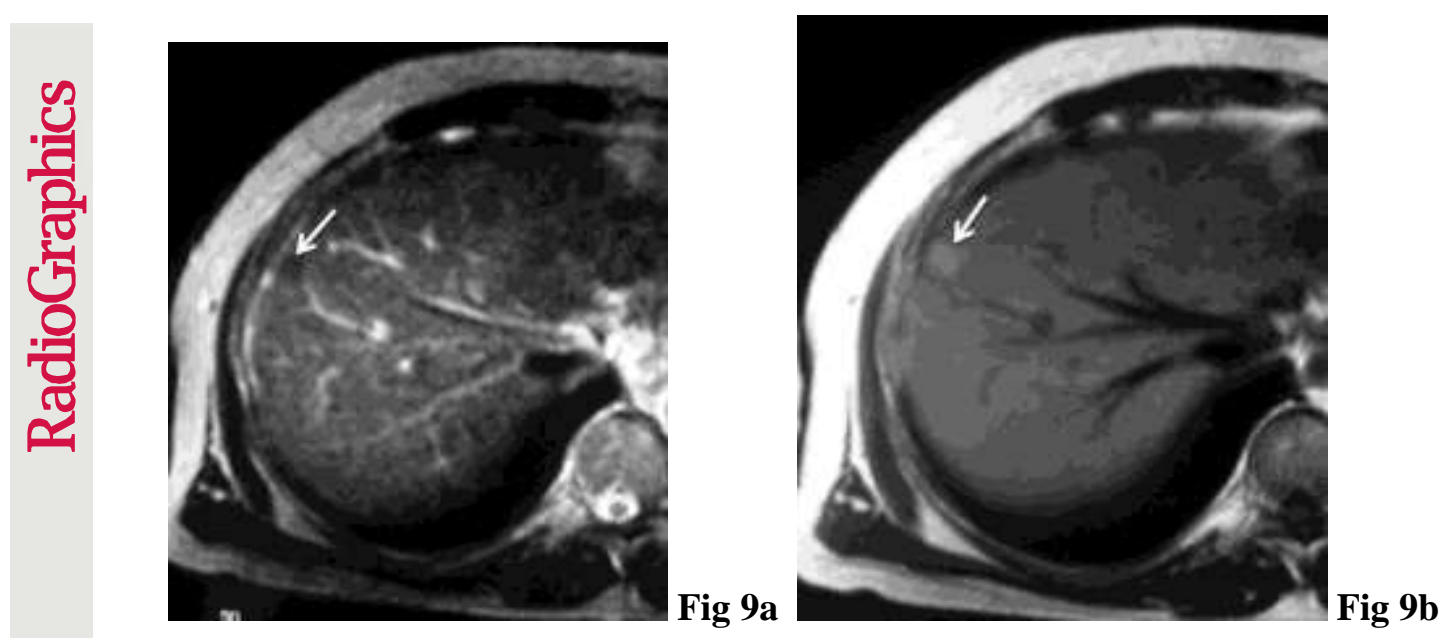

Figure 9. Dysplastic nodule in a 61-year-old man with alcoholic cirrhosis. (a, b) A small nodule in the right liver lobe (arrow) is (a) hypointense on T2-weighted (1800/93) image and (b) hyperintense on T1weighted (100/5.2) image. The low SI of the dysplastic nodule on the T2-weighted image may be related to deposition of paramagnetic substances such as iron. Note also several low-SI regenerative siderotic nodules on the T2-weighted image.

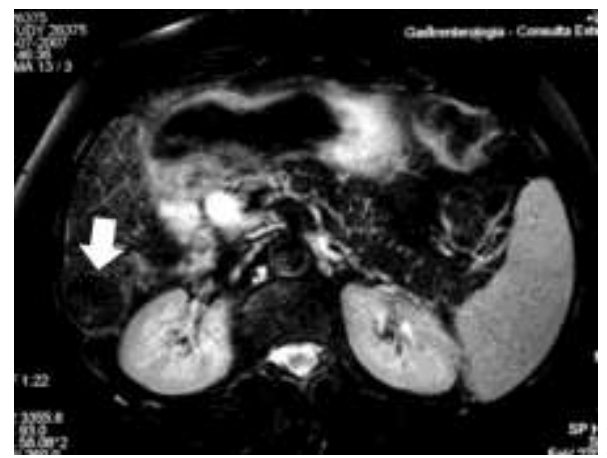

Fig 10a

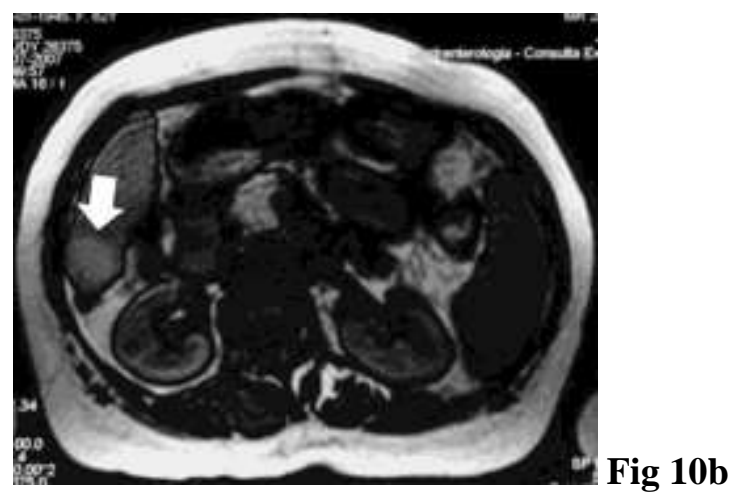

Figure 10. HCC in a 62-year-old woman with hepatitis $\mathrm{C}$ cirrhosis. (a, b) Focal lesion in the right liver lobe (arrow) is (a) hypointense on T2-weighted FSE image (3355/93) and (b) hyperintense on T1-weighted image (100/2.4). Low SI on T2-weighted images is related to abundant iron deposits within the tumor, as shown at pathologic study of the surgical specimen.
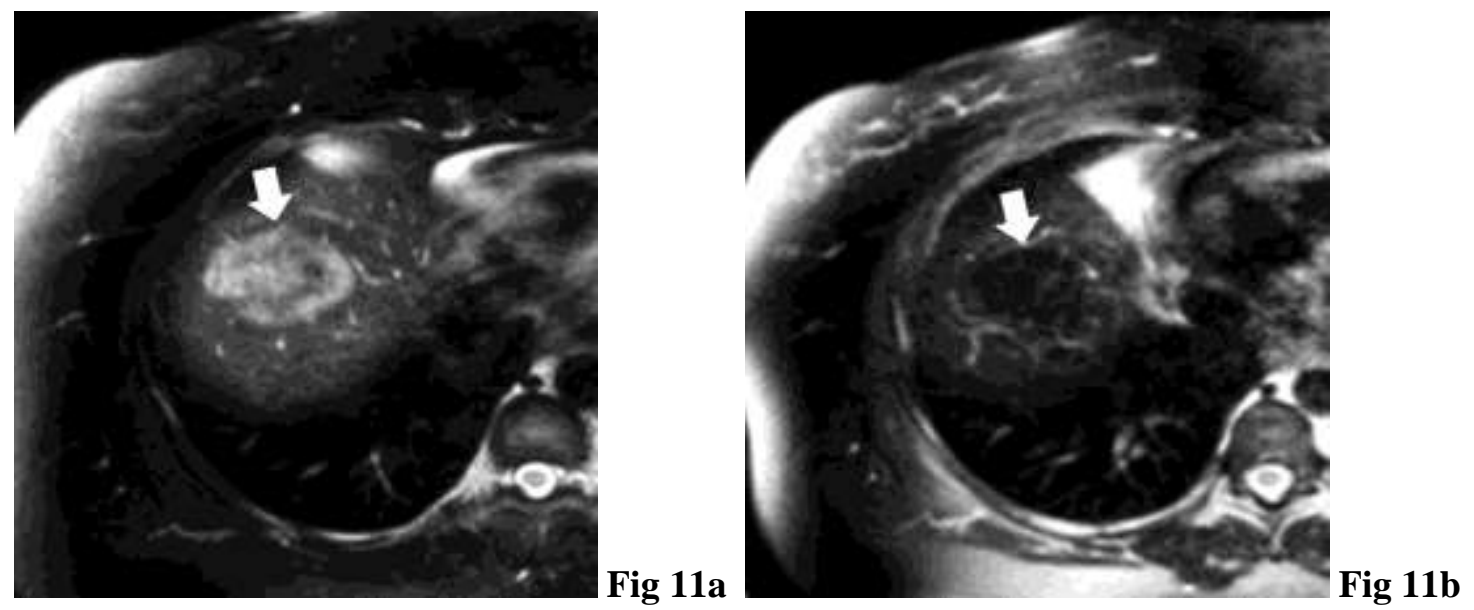
Figure 11. FNH in a 40-year-old woman. (a) A moderately hyperintense FNH (arrow) on the plain T2weighted FSE image (1800/93) becomes (b) hypointense relative to surrounding liver with the same sequence after intravenous administration of superparamagnetic iron oxides, owing to uptake of iron particles by Kupffer cells in the lesion. Contrary to the usually observed pattern of enhancement, this FNH shows greater SI loss than normal liver parenchyma.

\section{Copper}

There are two copper ions, monovalent copper and divalent copper. The former type is diamagnetic and does not have unpaired electrons (17); therefore, it does not influence the MR signal unless extremely high concentrations are achieved (18). Divalent copper is paramagnetic and decreases T1 and T2 relaxation rates, an effect due to one unpaired electron (17). However, divalent copper is usually bound to proteins, peptides, or amino acids. In normal liver, most of the stored copper is monovalent and bound to metallothionein $(17,19)$. Since the concentration of copper in liver is generally low in patients with Wilson disease, it may have no major effect on MR imaging, according to some authors (17). Nevertheless, multiple hypointense nodules on T2weighted MR images may be seen (20). These nodules have been ascribed to the paramagnetic effect of copper deposited in liver at a relatively early stage of the disease (21) (Fig 12). Because in later stages of the disease cirrhosis is present, the T2-weighted hypointensity seen in liver nodules has been related more to iron deposition (siderotic nodules) than to a real intranodular copper accumulation (22).
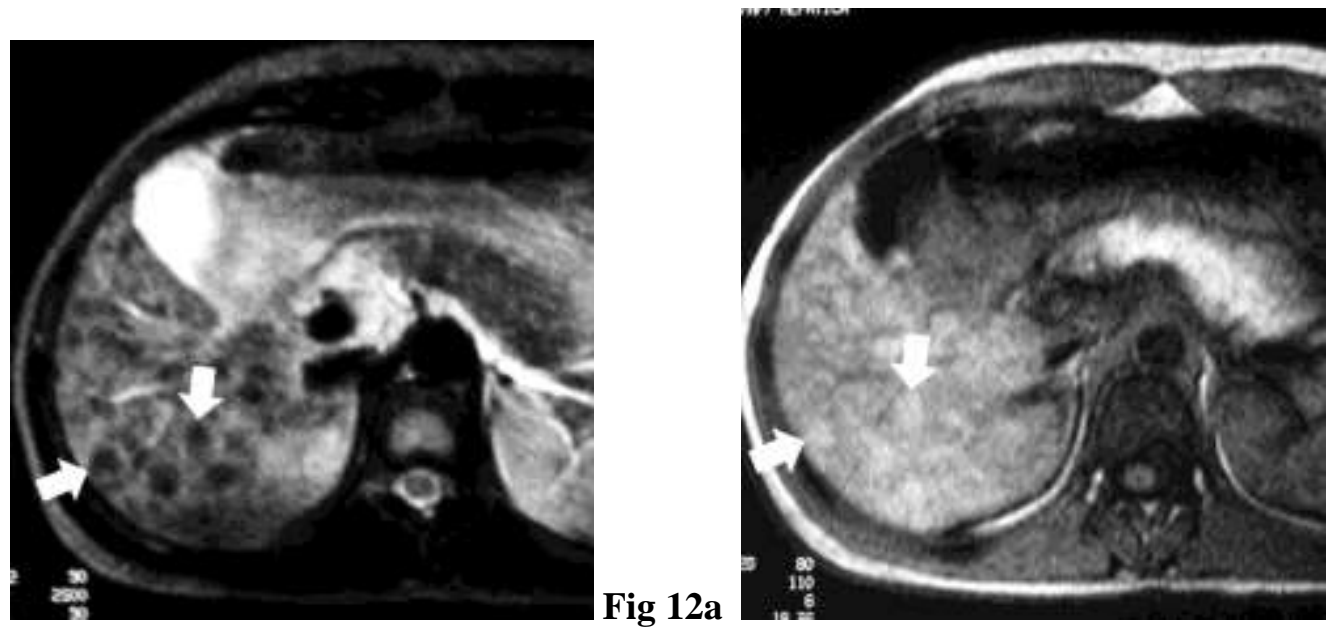

Fig 12b

Figure 12. Wilson disease in a 23-year-old woman. (a, b) Multiple liver nodules (arrows) are (a) hypointense on T2-weighted image (2300/90) and (b) hyperintense on T1-weighted image (110/6).

Early HCC may retain the property of copper uptake, a function that is progressively lost with tumor dedifferentiation (23). This uptake accounts for the hypointense appearance of HCC on T2-weighted images seen during the first steps of malignant transformation (Fig 13) (24). Also of note is that, rarely, accumulation of copper may occur within FNH in association with cholestatic features such as canalicular bile plugs (Fig 14) (25). 


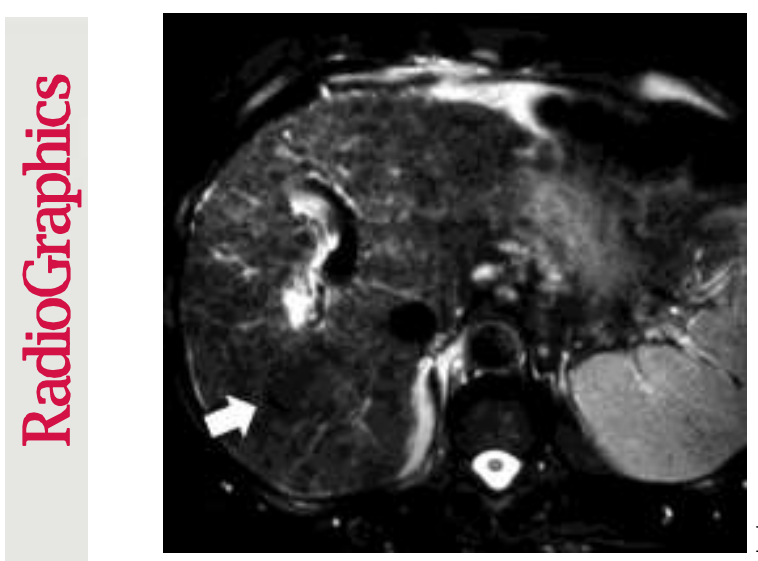

Fig 13a

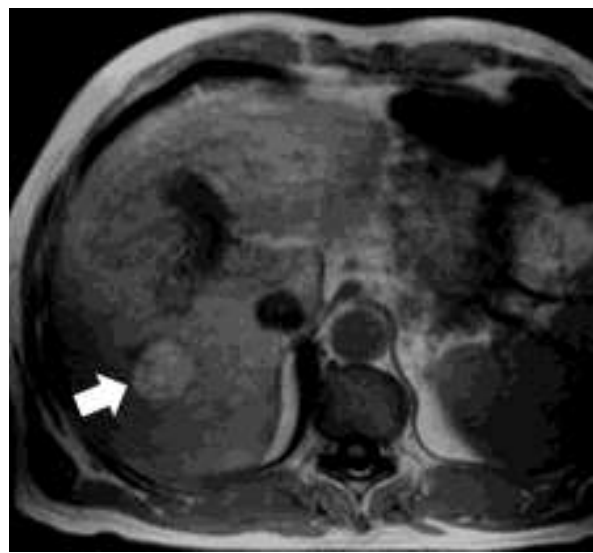

Fig 13b

Figure 13. Early-stage HCC in a 59-year-old man with alcoholic cirrhosis. (a, b) Focal lesion in the right liver lobe (arrow) demonstrates (a) low SI on T2-weighted FSE image (1800/93) and (b) hyperintensity on T1-weighted image (100/5.2). Low SI with the T2-weighted sequence was related to copper accumulation, as found at the pathologic study of the surgical specimen.
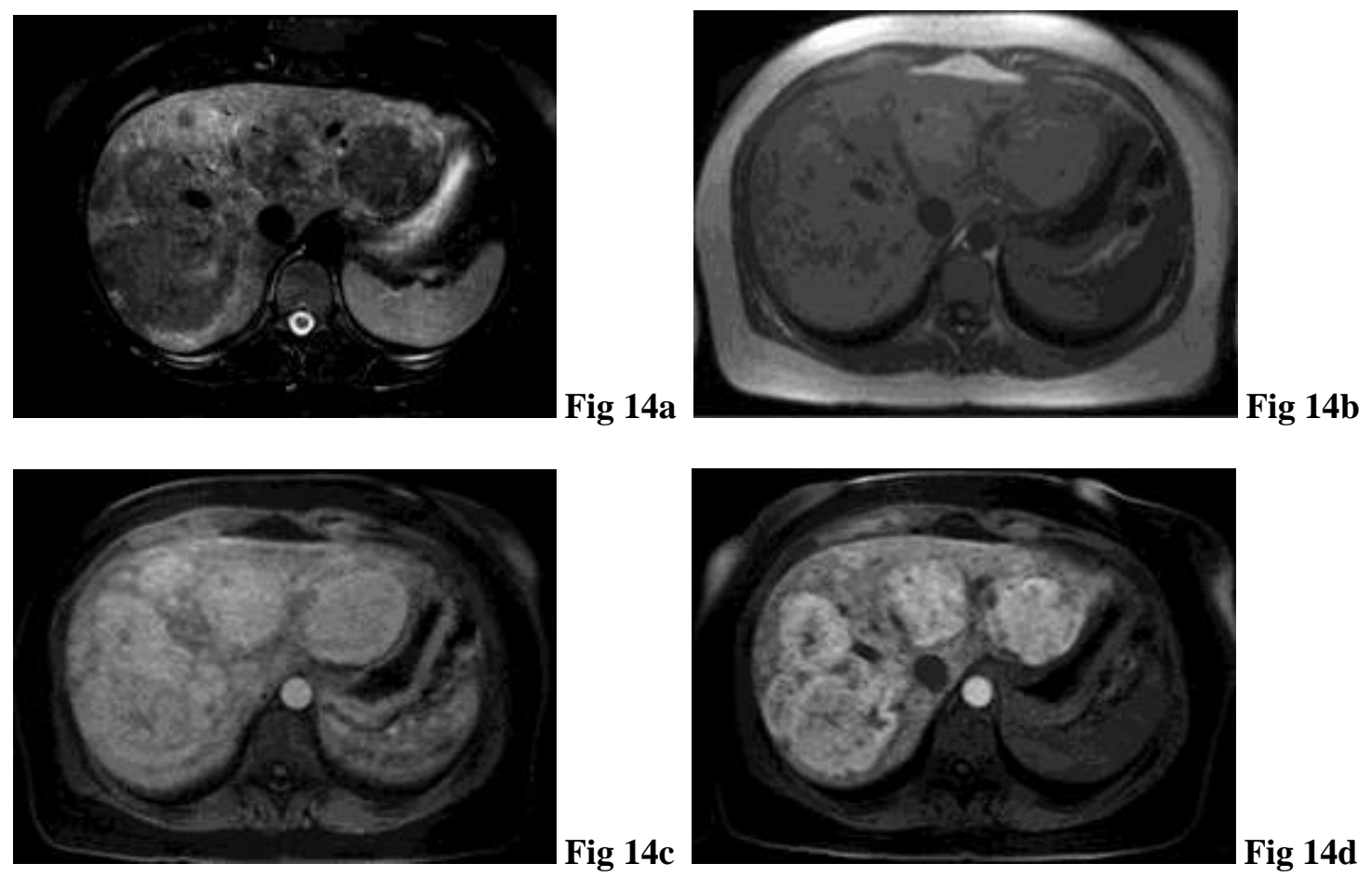

Figure 14. Multiple FNH in a 29-year-old woman. (a-d) Multiple FNH nodules are dispersed throughout the liver, (a) most of them hypointense on T2-weighted (1800/93) image, owing to the presence of copper, as proved by pathologic analysis with rhodamin staining. (b) The lesions have high SI on threedimensional T1-weighted FSE image (3.64/1.44) and (c) show hypervascular behavior after gadoxetate disodium injection. (d) During the hepatobiliary phase of this contrast agent, the lesions show marked enhancement. 
Teaching Point

\section{Macromolecules}

Free water molecules have unrestricted motion, whereas bound water (eg, associated with intracellular proteins) shows restricted motion. The hydrogen protons of bound water tumble with a frequency closer to the Larmor frequency, causing more efficient T1 and T2 relaxation. This results in a shortening of both the T1 and T2 relaxation times of water (26). Although this mechanism occurs to some extent in all tissues, some subtypes of soft tissue, such as muscle and fibrous tissue, are composed largely of macromolecules, which show this phenomenon to a greater extent. Magnetization transfer effects also contribute additively to lower the SI of macromolecule-rich tissues on T2-weighted images, especially when FSE techniques are used. The presence of multiple $180^{\circ}$ refocusing pulses increases off-resonance radiofrequency energy, contributing to the saturation of protons bound to macromolecules (26).

\section{Smooth Muscle}

Compared to other tissues in the body, both smooth and skeletal muscle have low SI on T2weighted images, owing to the T2-shortening effects of intramuscular actin, myosin, and collagen and decreased extracellular fluid $(27,28)$. Because of the predominance of smooth muscle, angiomyolipoma and hepatic leiomyoma are examples of hypointense focal lesions on T2weighted images (Fig 15).

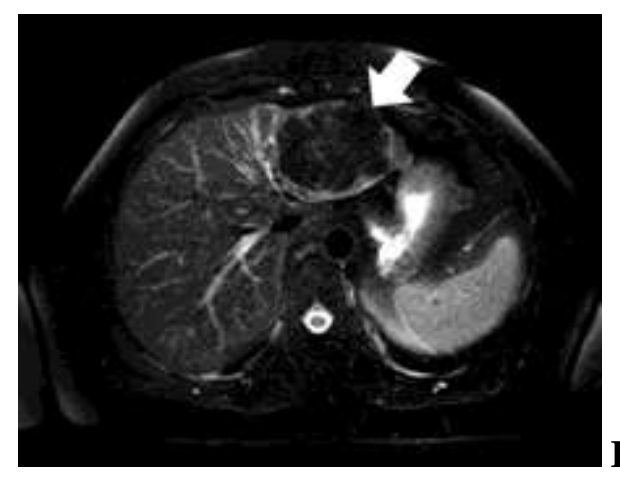

Fig 15a
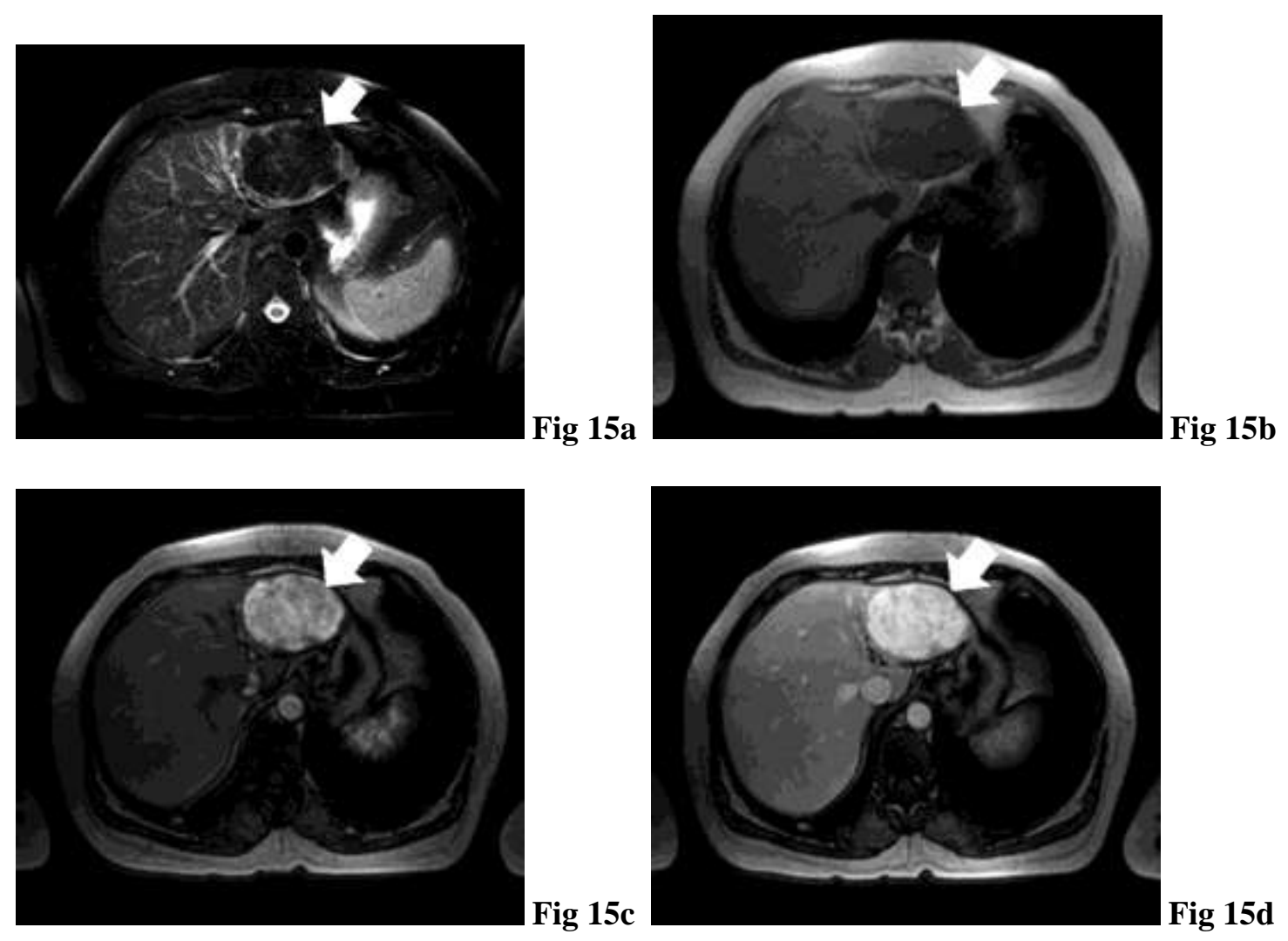

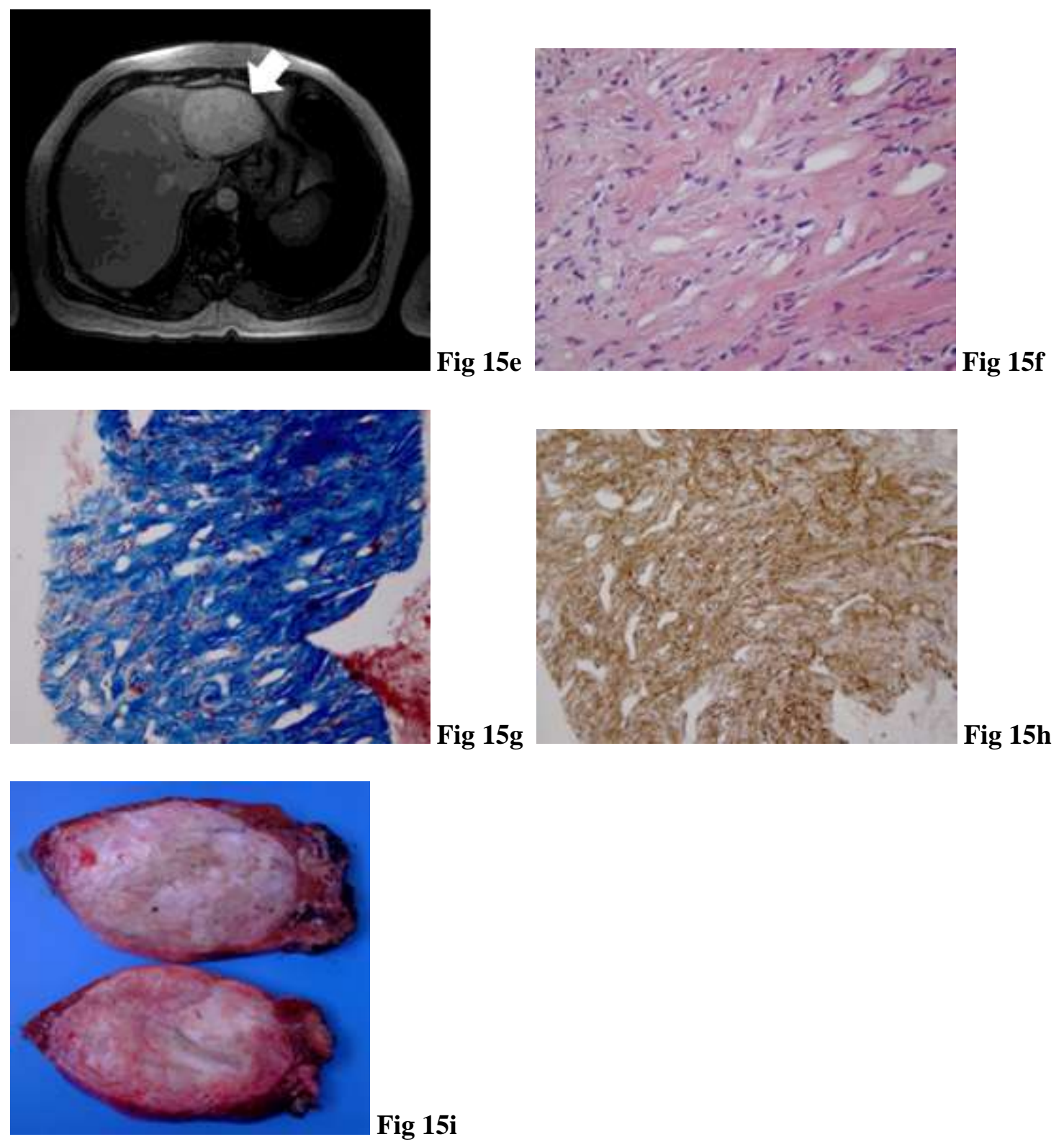

Figure 15. Leiomyoma in a 49-year-old woman. (a) T2-weighted FSE image (1800/93) depicts a low-SI mass (arrow) in the left liver lobe, which (b) is also hypointense on T1-weighted image (100/2.4). (c-e) On the images from the dynamic study, the mass shows a marked progressive enhancement throughout the liver enhancement phases. (f) High-power photomicrograph (original magnification, $\times 200$ ) shows smooth muscle fibers without atypia. Histochemical studies are positive for (g) trichrome (original magnification, $\times 200$ ) and (h) smooth muscle actin (original magnification, $\times 200$ ). (i) Pathologic specimen of the left liver lobe mass confirms a well-defined lesion.

\section{Fibrosis}

Paucicellular regions of mature fibrous tissue (with a large proportion of collagen and few cells and vessels) display, like compact smooth muscle, low SI on T2-weighted images (26). Various amounts of fibrous tissue are commonly present in several tumors. In fibrolamellar carcinoma, the tumors are formed by abundant fibrosis arranged in bands, or laminae $(29,30)$, which explain their hypointense appearance (Fig 16). The presence of substantial amounts of fibrosis in cholan- 
giocellular carcinoma explains the presence of the low-SI areas seen on T2-weighted images (Fig 17) $(8,9)$.

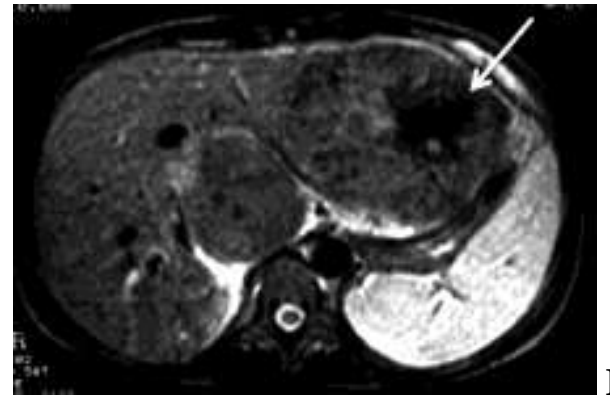

Fig 16a

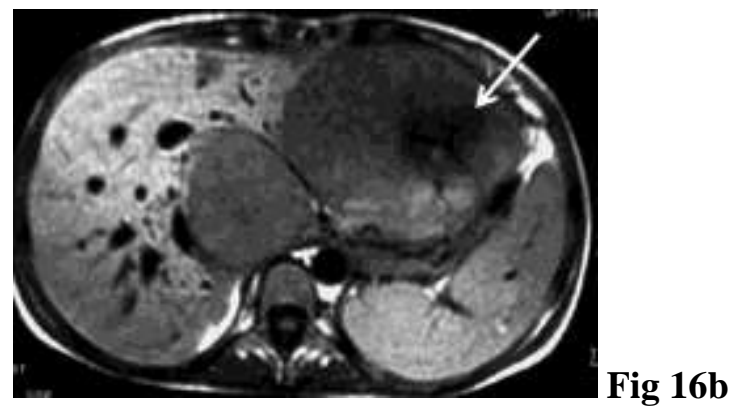

Figure 16. Fibrolamellar carcinoma in a 57-year-old man. (a, b) Focal lesion (arrow) in the left liver lobe shows low SI on (a) T2-weighted FSE image (1800/93) and (b) T1-weighted image (680/6). The lesion has a central hypointense scar with both sequences, owing to fibrotic content and calcification. Note the additional tumor in the caudate lobe.
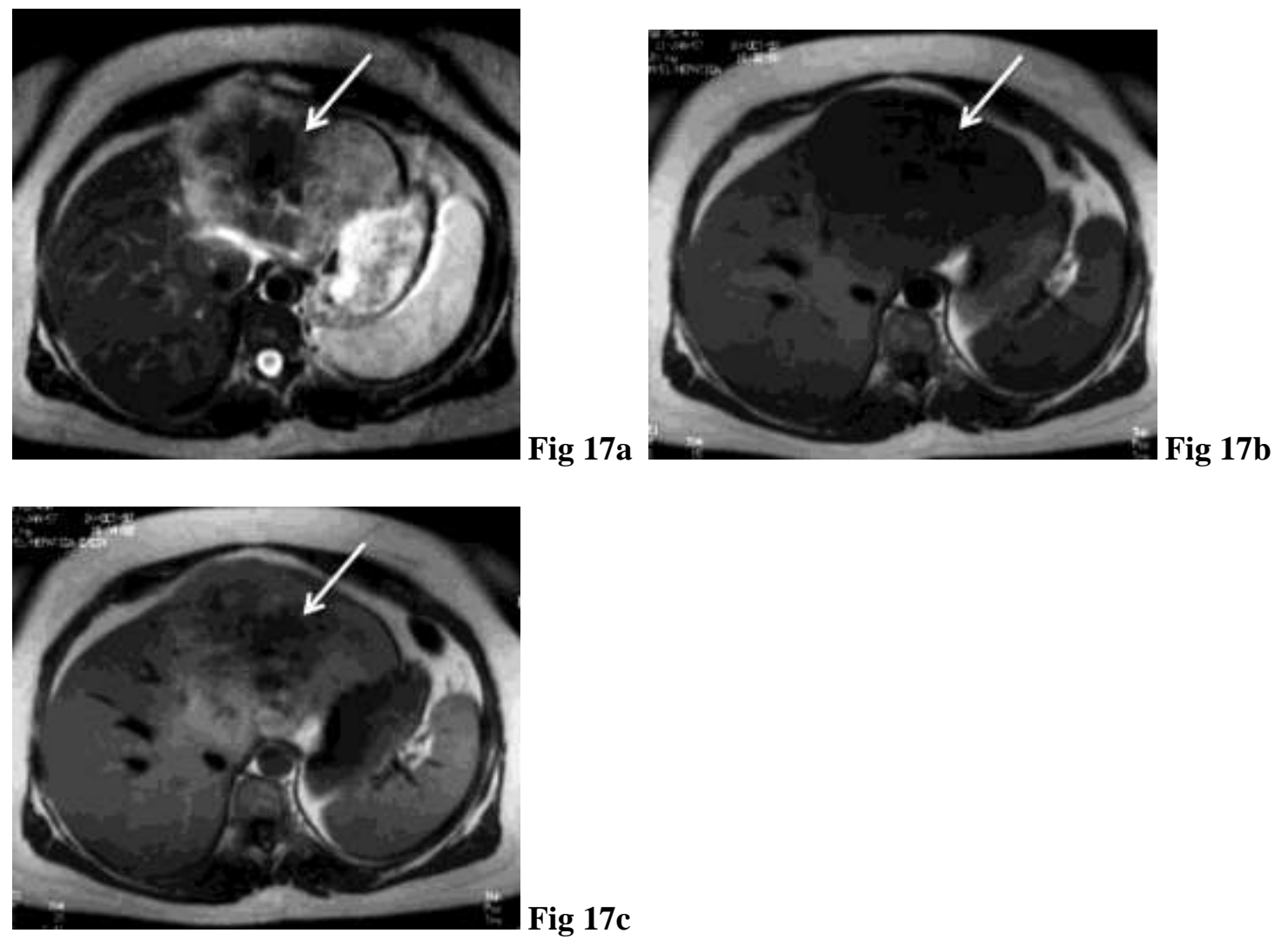

Figure 17. Intrahepatic cholangiocarcinoma in a 40-year-old woman. (a, b) Large mass (arrow) in the left liver lobe with (a) an extensive central area of low SI on T2-weighted (1800/93) image, corresponding to a fibrous core. (b) The lesion is quite hypointense on T1-weighted image (704/15). (c) On the delayedphase image obtained about 30 minutes after injection, heterogeneous peripheral enhancement with a gadolinium-based contrast agent is seen.

Metastases with a large amount of fibrous noncalcified matrix (especially due to adenocarcinoma) can also show T2-weighted hypointensity (Fig 18) (9). Liver lesions in which cellularity is 
progressively replaced by fibrosis may display low SI on T2-weighted images, as is the case in treated liver lymphoma or healed inflammatory lesions (Fig 19).

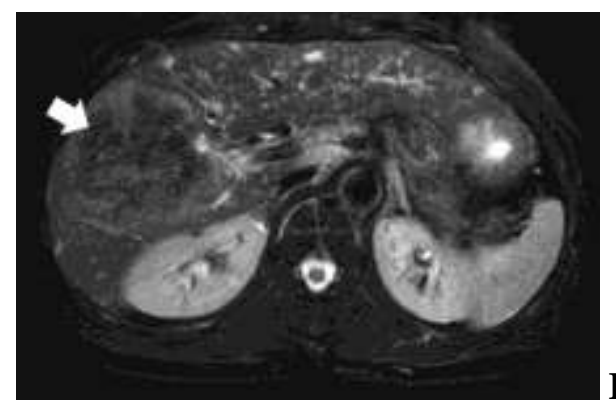

Fig 18a

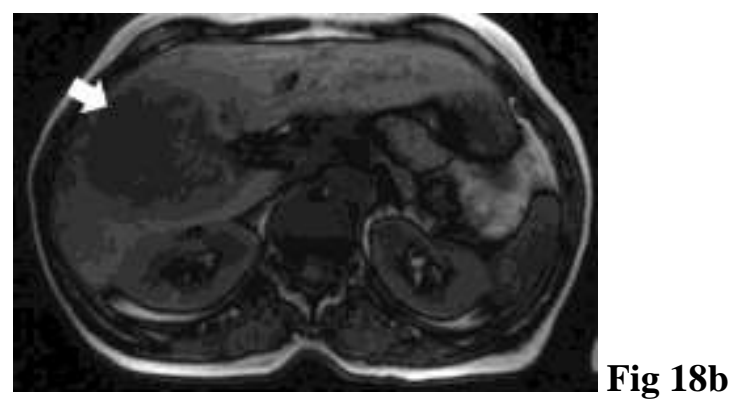

Figure 18. Metastasis in a 72-year-old man with bronchogenic adenocarcinoma. $(\mathbf{a}, \mathbf{b})$ Large mass in the right liver lobe (arrow) shows (a) predominant low SI on T2-weighted FSE image (1800/93), corresponding to central fibrosis in the biopsy specimen. (b) The lesion is also hypointense on T1-weighted image $(100 / 2.4)$.

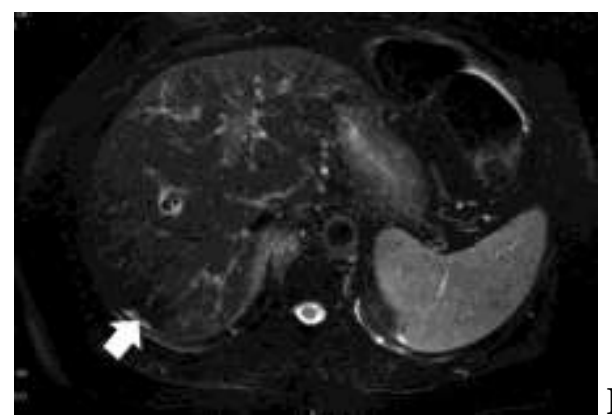

Fig 19a

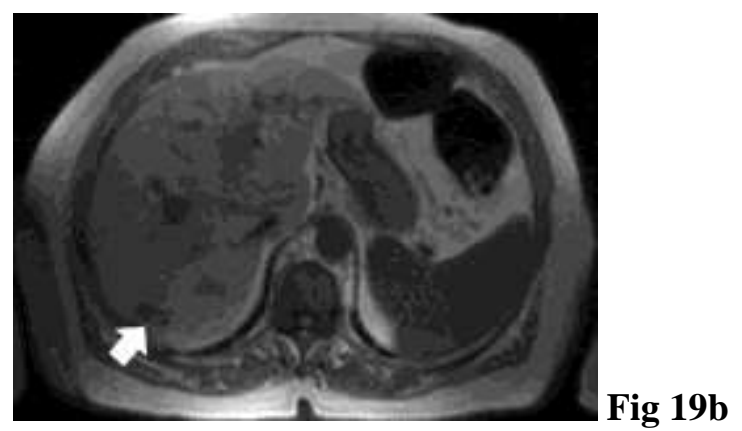

Figure 19. Healed abscess in a 45 -year-old woman. (a, b) A peripherally located, ill-defined liver lesion (arrow) shows (a) low SI on T2-weighted FSE image (1800/93) and (b) T1-weighted image (100/5.2). This hypointensity is related to fibrosis due to a healed abscess.

\section{Mucin}

Mucin contains carbohydrate-rich glycoproteins. Hydrated mucinous tissue has high SI on T2weighted images; however, in desiccated mucinous secretions, the decrease in free water can result in a marked $\mathrm{T} 2$ shortening, which can be so pronounced that it results in very low SI on T2-weighted images (31).

Owing to their mucin content, hepatic metastases from colorectal, gastric, pancreatic, or ovarian cancers appear dark on T2-weighted images, resulting in the so-called cauliflower appearance.

\section{Melanin}

Metastatic melanoma to the liver has variable imaging features that depend on the melanin content of metastases, which causes different hyperintense/hypointense patterns on T1- and T2weighted images (32). Usually, on T2-weighted images high signal intensity is seen in larger metastatic lesions, whereas smaller lesions may appear isointense (33). In some instances, however, metastases from malignant melanoma cause T2 shortening, presumably related to higher melanin levels (or to old hemorrhage), ultimately leading to T2-weighted hypointensity (Fig 20) $(8,9)$. 

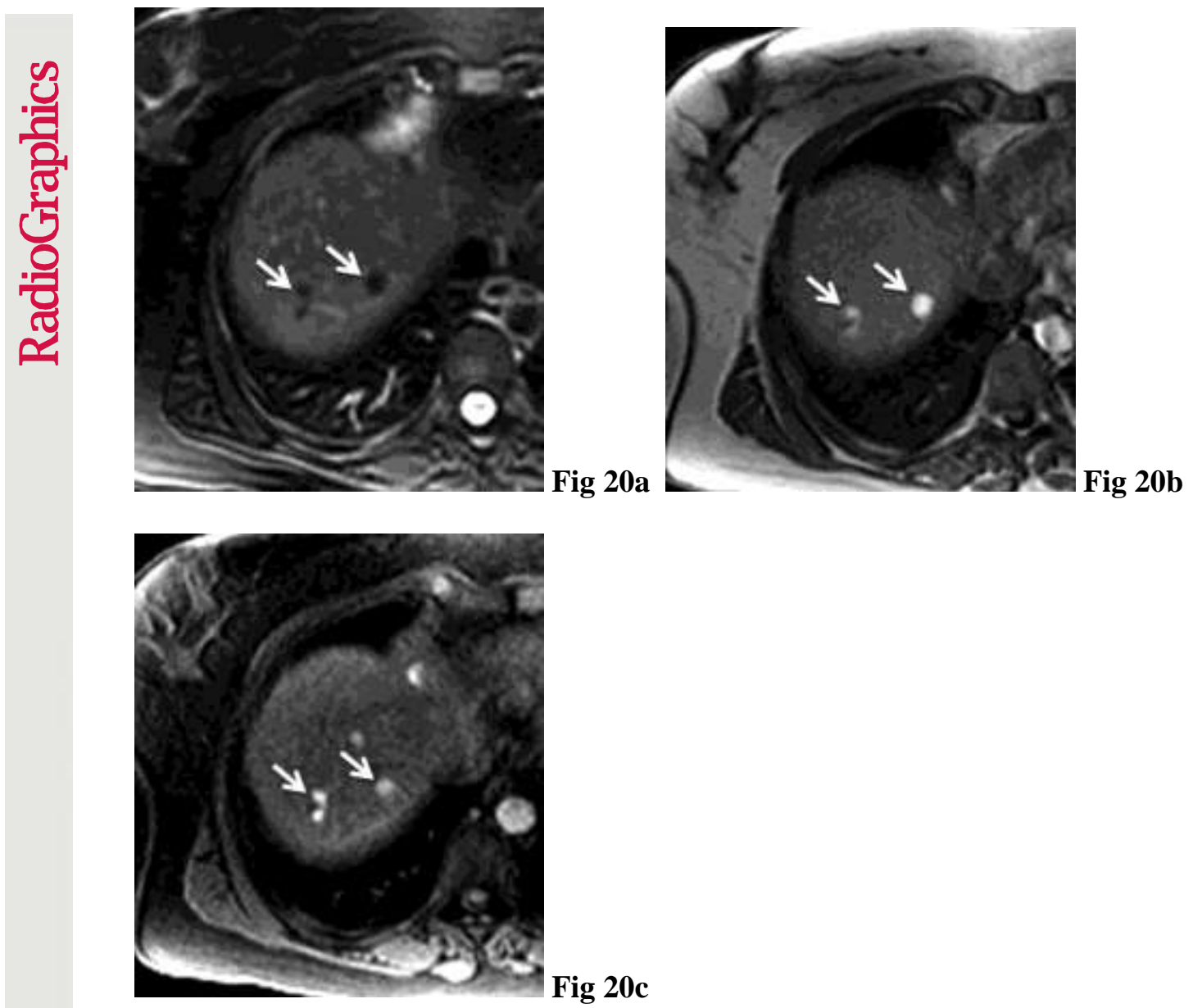

Figure 20. Metastatic melanoma in a 53-year-old man. (a-c) Multiple liver nodules (arrows) are visible, (a) hypointense on T2-weighted image (1800/93) and hyperintense on both the (b) conventional T1weighted image (692/10) and (c) T1-weighted FSE image.

\section{Fibrinogen}

Fibrinogen has also been indicated as another possible cause of hypointensity on T2-weighted images (34). Pale bodies, which are intracytoplasmic inclusions, represent accumulations of fibrinogen in dilated rough endoplasmic reticulum and correlate with the sclerotic changes reported in cases of HCC and with areas of low SI seen on T2-weighted images (34).

\section{Necrosis}

Necrotic areas may exhibit variable features on MR images, since liquefactive necrosis is highly hyperintense on T2-weighted images, owing to increased water content, whereas coagulative (dehydrated) necrosis is characterized by low SI on T2-weighted images (9). This explains the T2-weighted appearance of necrosis seen in some focal liver lesions, especially those resulting from percutaneous ablative therapies such as radiofrequency ablation (Fig 21) $(9,35,36)$. 

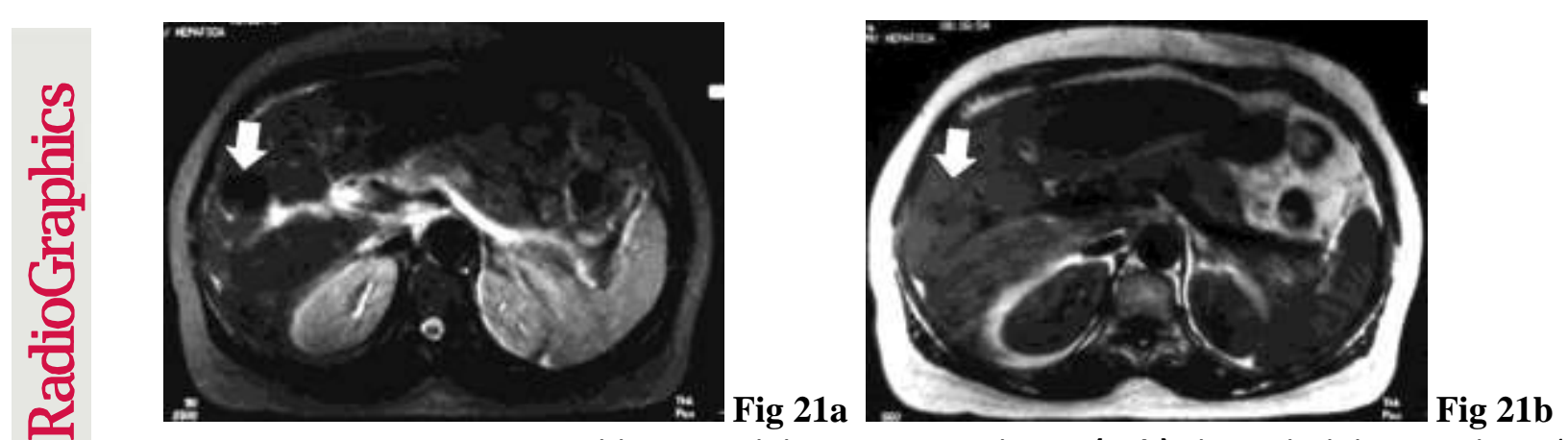

Figure 21. HCC in a 71-year-old man with hepatitis $\mathrm{C}$ cirrhosis: $(\mathbf{a}, \mathbf{b})$ This right lobe neoplasm (arrow) shows (a) low SI on T2-weighted image (2300/90), owing to previous therapy with radiofrequency thermal ablation. (b) The lesion is only slightly hyperintense on T1-weighted image (680/15).

As recently described, the solitary necrotic nodule in the liver is an uncommon nonmalignant entity of unknown cause that displays T2-weighted hypointensity. The explanation for this curious finding is thought to be related to its low level of hydration, vascularity, and cellularity and to the presence of coagulative necrosis (Fig 22) (37).

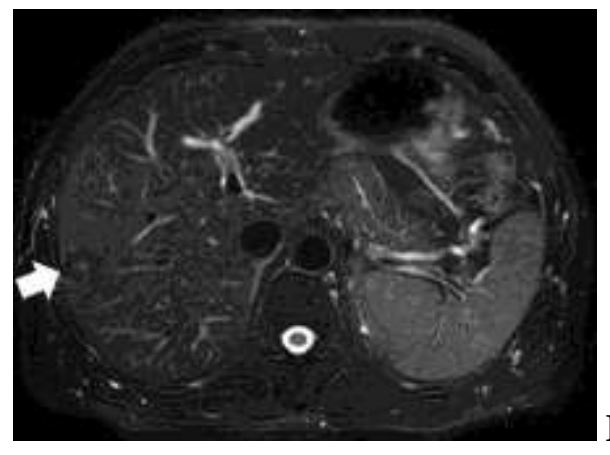

Fig 22a

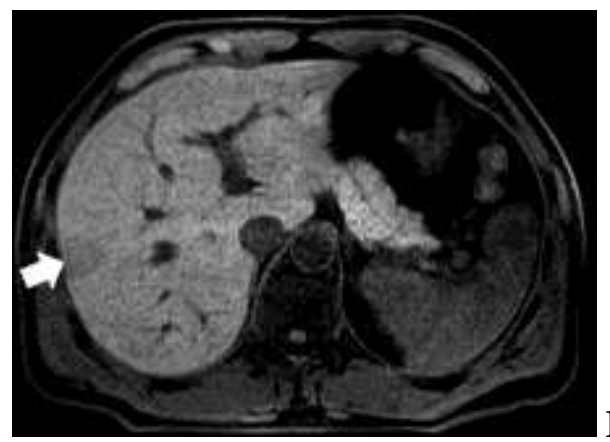

Fig 22b

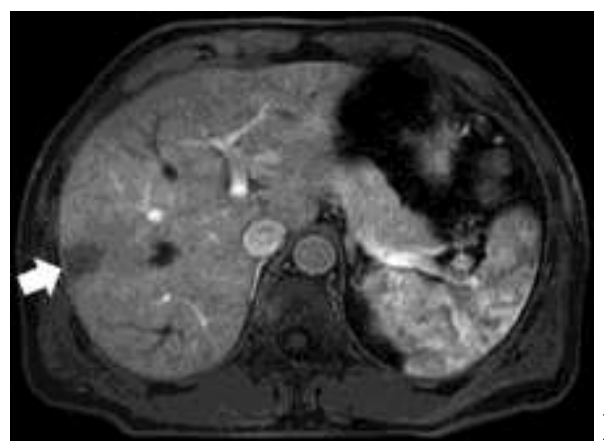

Fig 22c

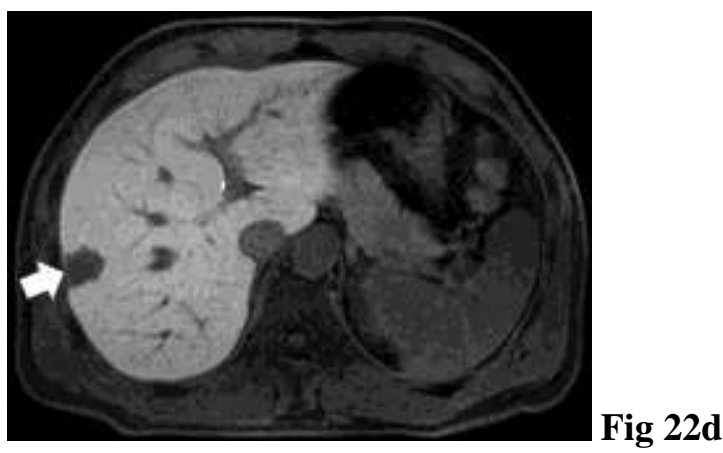

Figure 22. Solitary necrotic nodule in the liver of a 33-year-old woman. (a-d) This right lobe nodule (arrow) is hypointense on both the T2-weighted image (1800/93) and the three-dimensional T1-weighted FSE image (3.64/1.44). After gadoxetate disodium administration, the nodule remains hypointense during the (c) late arterial phase (indicating hypovascularity) and (d) the hepatobiliary phase.

\section{Calcium}

Calcification typically has low SI with both T1-weighted and T2-weighted sequences; however, faint or tiny calcifications may not be detectable on MR images (38). Extensively calcified lesions such as granulomas (Fig 23) or healed inactive hepatic hydatid cysts (Fig 24) appear hypointense on T2-weighted images (38). Areas of calcification in other lesions may also be found, 
as is the case in HCC, especially in its fibrolamellar variant, owing to central scar calcification (39). Some metastases also have calcifications and consequently low SI on T2-weighted images (29). This has been reported in metastases from mucinous cancers of the colon, stomach, breast, and ovary, as well as in cases of calcified-matrix-producing tumors such as osteosarcomas and chondrosarcomas (38). The rare hepatic teratoma may also show hypointense, calcified foci of variable size, as in other areas of the body (Fig 25) (40).

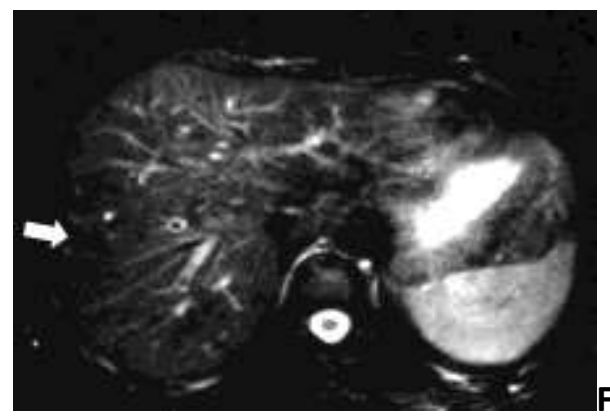

Figure 23. Granuloma in a 42-year-old woman with previous tuberculous infection of the lungs. A small focal lesion (arrow) in the right liver lobe, corresponding to a calcified granuloma, shows low SI on T2-weighted FSE image (2300/90).

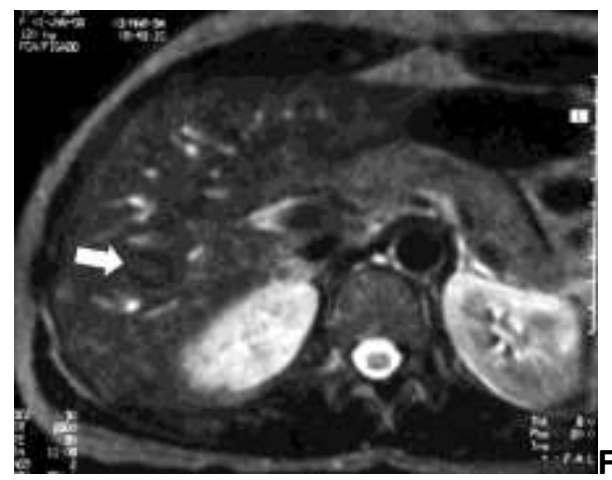

Figure 24. Hydatid cyst in 48-year-old woman with hepatic echinococcosis. A focal lesion (arrow) in the right lobe of the liver, corresponding to a calcified, inactive hydatid cyst, is hypointense on T2-weighted image (2300/90).
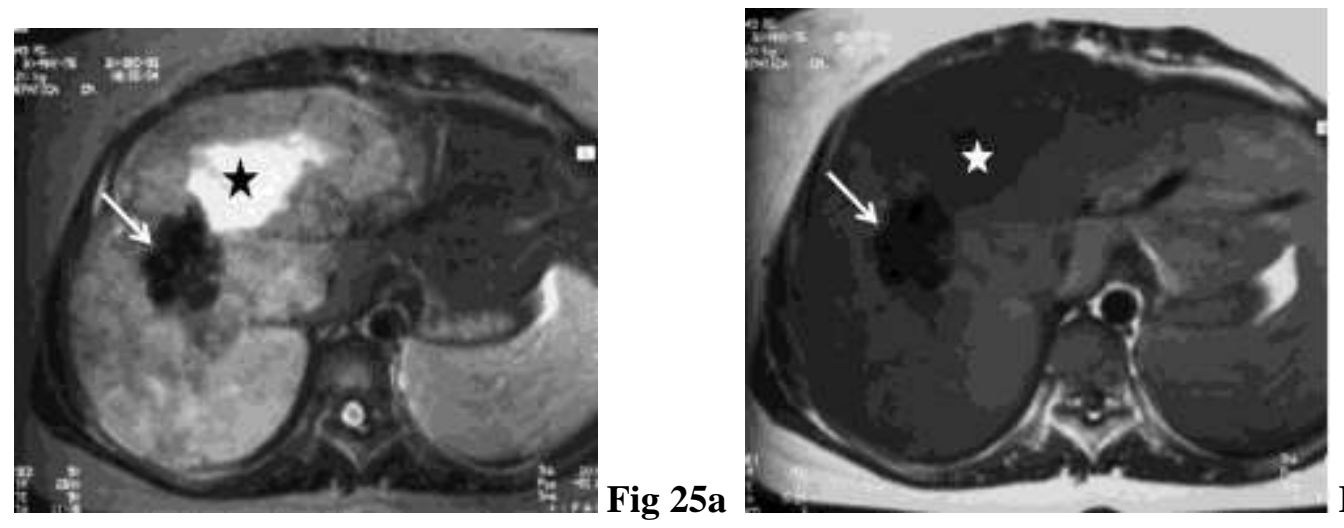

Fig 25b

Figure 25. Teratoma in a 17-year-old girl. (a, b) This rare hepatic lesion (arrow) may present with internal areas of calcification, which have very low SI on both the (a) T2-weighted image (2300/90) and the (b) T1-weighted image (750/17). Note also an internal necrotic area ( $\star$ ).

\section{Fat}


Fat has intermediate SI relative to muscle on conventional T2-weighted images and has high SI relative to muscle on FSE T2-weighted images. The latter sequence minimizes the signal loss in fat due to the dephasing effects of J-coupling and magnetization transfer. Currently, T2-weighted FSE sequences are regularly used in the majority of abdominal protocols, since these protonselective sequences decrease potential artifacts due to subcutaneous and intraabdominal fat. An additional reason for this approach is the ability to rapidly distinguish between fat and hemorrhage. Therefore, even though MR protocols usually rely on FSE T1-weighted and T1-weighted in- and out-of-phase sequences for characterization of fatty lesions, it is also possible to affirm that if a mass loses SI when a T2-weighted sequence is applied, a macroscopic fat component is present. In cases of fatty metamorphosis within hepatocellular adenoma (Fig 26) (41) or HCC (Fig 27), intratumoral fat may be hypointense on FSE T2-weighted images (40). This observation also applies to the diagnosis of fatty tumors such as angiomyolipoma (Fig 28) or lipoma (40). Fat infiltration of FNH is exceedingly rare (42) and is thought to be an extension of the patient's steatosis or result from ischemia due to tumoral compression of adjacent liver. In these cases, FNH may appear hypointense on FSE T2-weighted images $(43,44)$.
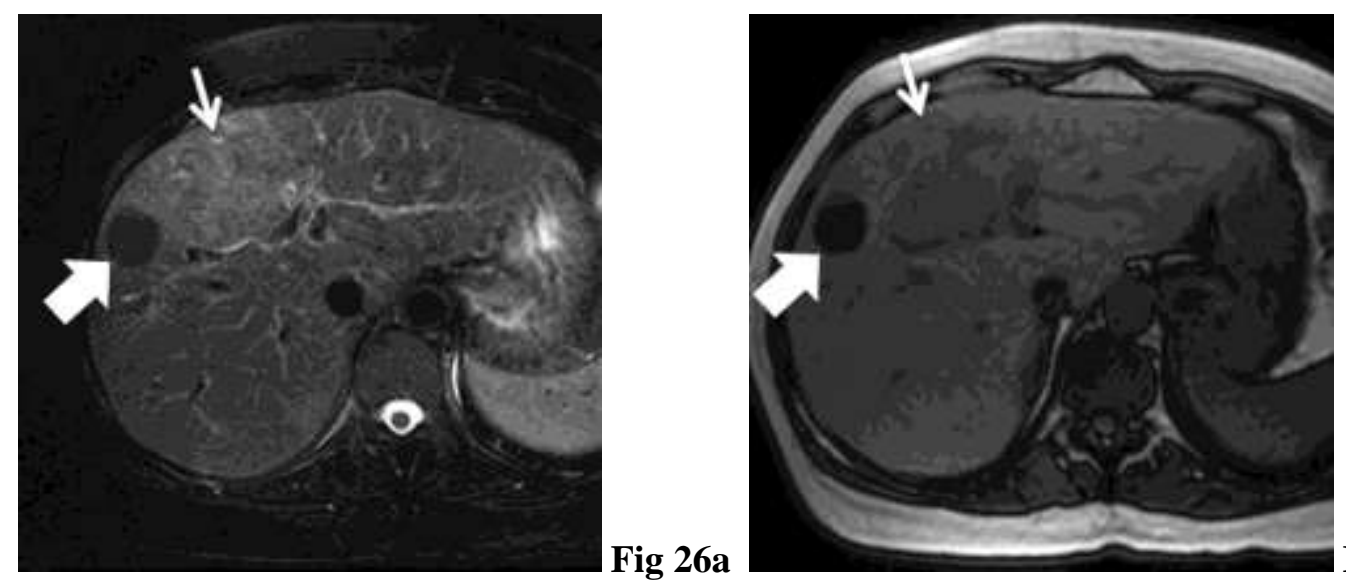

Fig 26b

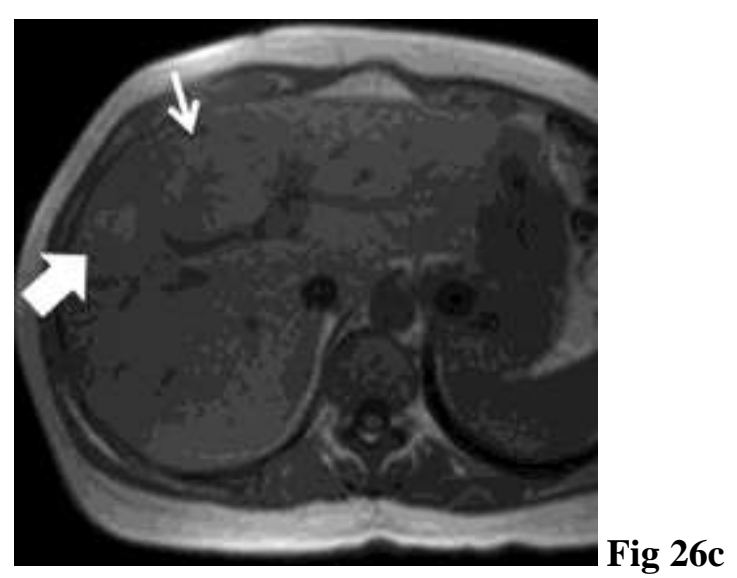

Figure 26. Steatotic type of hepatocellular adenoma in a 38-year-old woman. Sharply marginated nodule (thick arrow), hypointense on (a) T2-weighted FSE image (1800/93) and (b) T1-weighted out-of-phase image (100/2.4) and (c) hyperintense on T1-weighted in-phase image (100/5.2), findings indicative of fatty metamorphosis. Note the large FNH nearby (thin arrow). 

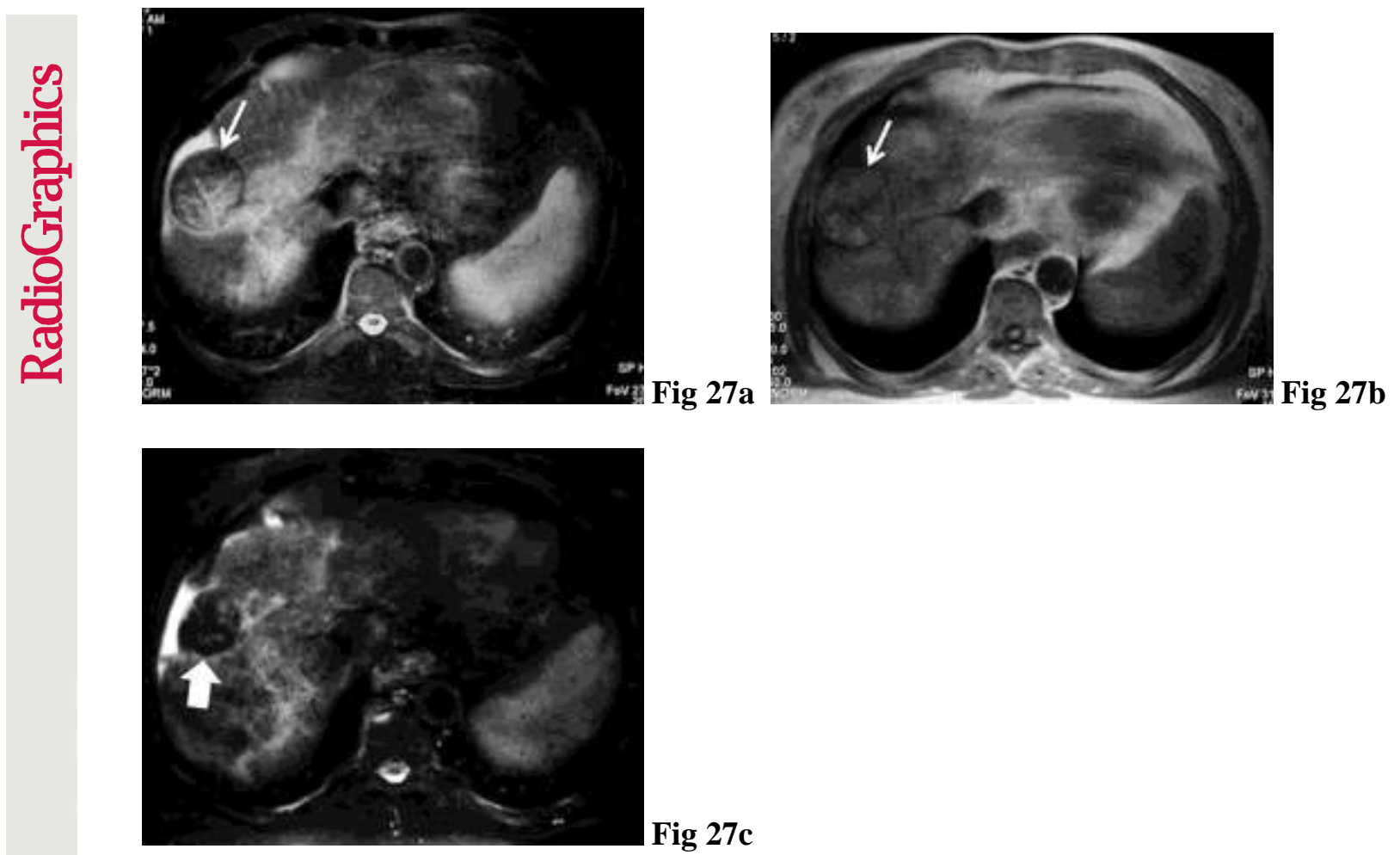

Figure 27. HCC in an 80-year-old man with alcoholic cirrhosis. (a-c) The capsulated tumor (arrow) demonstrates a heterogeneous appearance with (a) extensive low-SI zones on the T2-weighted FSE image (4944/94), which correspond to (b) high-SI areas on the T1-weighted image (200/4.8), indicating the presence of fat. (c) A few months after radiofrequency thermal ablation, the neoplasm is almost totally hypointense on T2-weighted image as a result of coagulative necrosis.

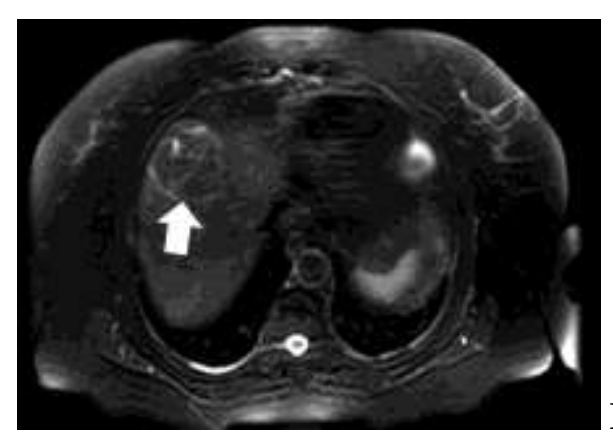

Fig 28a
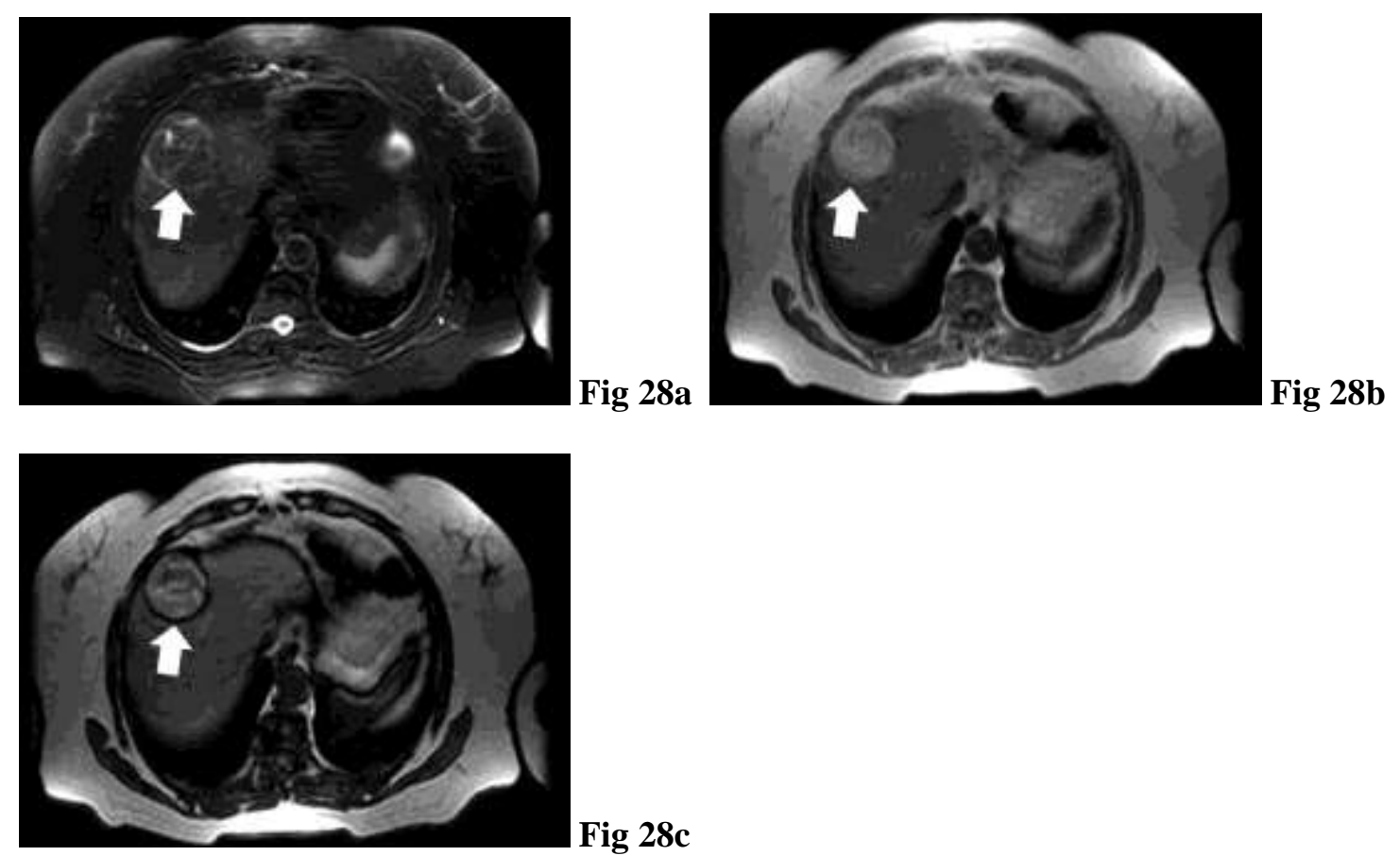

Fig 28c

Figure 28. Angiomyolipoma in a 66-year-old man. (a-c) The lesion (arrow) is (a) hypointense on T2weighted FSE image (1800/93) and (b) hyperintense as subcutaneous fat on in-phase T1-weighted image 
(100/5.2). (c) Note the India ink artifact around the lesion on the T1-weighted out-of-phase image $(100 / 2.4)$, owing to chemical shift artifact at the boundary between fat within the tumor and adjacent liver parenchyma.

\section{SUMMARY}

Hypointensity on T2-weighted images, although a less common presentation of a wide spectrum of focal liver lesions (both benign and malignant), has a variety of causes. Knowledge of these causes is helpful in deriving the correct diagnosis, but it should be integrated in the proper clinical context. In more complex cases, however, radiologic-pathologic correlation is necessary to understand the complete picture of hypointense appearances.

\section{References}

1. Hussein SM. Hemorrhage. In: Hussein SM, ed. Liver MR imaging. Berlin, Germany: Springer, 2007;28-29.

2. Grazioli L, Federle MP, Brancatelli G, Ichikawa T, Olivetti L, Blachar A. Hepatic adenomas: imaging and pathologic findings. Radiographics 2001;21(4):877-892.

3. Ferlicot S, Kobeiter H, Nhieu JTV, et al. MR imaging of atypical focal nodular hyperplasia of the liver: radiology-pathology correlation. AJR Am J. Roentgenol 2004;182:1227-1231.

4. Brancatelli G, Federle MP, Grazioli L, Golfieri R, Lencioni R. Benign regenerative nodules in Budd-Chiari syndrome and other vascular disorders of the liver: radiologic-pathologic and clinical correlation. Radiographics 2002;22(4):847-862.

5. Kim T, Baron RL, Nalesnik MA. Infarcted regenerative nodules in cirrhosis: CT and MR imaging findings with pathologic correlation. AJR Am J Roentgenol 2000;175:1121-1125.

6. Bonkovsky HL. Iron and the liver. Am J Med Sci 1991;301:32-43.

7. Saokar A, Braschi M, Harisinghani M. Lymphotrophic nanoparticle enhanced MR imaging (LNMR imaging) for lymph node imaging. Abdom Imaging 2006;31:660-667.

8. Bartolozzi C, Lencioni R, Donati F, Cioni D. Abdominal MR: liver and pancreas. Eur Radiol 1999;9(8):1496-1512.

9. Martí-Bonmatí L. MR imaging characteristics of hepatic tumors. Eur Radiol 1997;7(2):249258.

10. Terada T, Kadoya M, Nakanuma Y, Matsui O. Iron-accumulating adenomatous hyperplastic nodule with malignant foci in the cirrhotic liver: histopathologic, quantitative iron, and magnetic resonance imaging in vitro studies. Cancer 1990;65:1994-2000.

11. Williams GM. The pathogenesis of rat liver cancer caused by chemical carcinogens. Biochem Biophys Acta (CR) 1980;605:167-189.

12. Beaumont C, Le Rumeur E, Guillouzo C, et al. Ferritin is increased in diethylnitrosamine altered rat hepatocytes. Biochem Biophys Res Commun 1981;99:879-885.

13. Lescoat G, Loreal O, Moirand R, et al. Iron induction of ferritin synthesis and secretion in human hepatoma cell (HepG2) cultures. Liver 1989;9:179-185.

14. Honda H, Kaneko K, Kanazawa Y, et al. MR imaging of hepatocellular carcinomas: effect of $\mathrm{Cu}$ and Fe contents on signal intensity. Abdom Imaging 1997;22(1):60-66. 
15. Vogl TJ, Hammerstingl R, Schwarz W. Superparamagnetic iron oxide-enhanced versus gadolinium-enhanced MR imaging for differential diagnosis of focal liver lesions. Radiology 1996;198:881-887.

16. Semelka RC, Helmberger TKG. Contrast agents for MR imaging of the liver. Radiology 2001;218:27-38.

17. Kitagawa K, Matsui O, Kadoya M, et al. Hepatocellular carcinomas with excessive copper accumulation: CT and MR findings. Radiology 1991;180:623-628.

18. Nakakoshi T. Copper and hepatocellular carcinoma. Radiology 2000;214(1):304-306.

19. Bremner I. Involvement of metallothionein in the hepatic metabolism of copper. J Nutr 1987;117:19-29.

20. Akhan O, Akpinar E, Karcaaltincaba M, et al. Imaging findings of liver involvement of Wilson's disease. Eur J Radiol 2009;69:147-155.

21. Ko S, Lee T, Ng S, Lin J, Cheng Y. Unusual liver MR findings of Wilson's disease in an asymptomatic 2-year-old girl. Abdom Imaging 1998;23:6-9.

22. Ito K, Hussain SM, Mitchell DG, Semelka RC. Diffuse liver disease. In: Edelman RR, Hesselink JR, Zlatkin MB, Crues III JV, eds. Clinical magnetic resonance imaging. 3rd ed. Philadelphia, Pa: Saunders, 2006;2589-2631.

23. Haratake J, Horie A, Takeda S, et al. Tissue copper content in primary and metastatic liver cancers. Acta Pathol Jap 1987;37:231-238.

24. Ebara M, Watanabe S, Kita K, et al. MR imaging of small hepatocellular carcinoma: effect of intratumoral copper content on signal intensity. Radiology 1991;180:617-621.

25. Sternlieb I. Copper and the liver. Gastroenterology 1980;78:1615-1628.

26. Seigelman ES, Outwater EK. Tissue characterization in the female pelvis by means of MR imaging. Radiology 1999;212:5-18.

27. Misra LK, Kim EE, Hazlewood CF, Todd LE, Elizondo-Riojas G. Characterization of skeletal muscles by MR imaging and relaxation times. Physiol Chem Phys Med NMR 1990;22:219-228.

28. Polak JF, Jolesz FA, Adams DF. NMR of skeletal muscle: differences in relaxation parameters related to extracellular/intracellular fluid spaces. Invest Radiol 1988;23:107-112.

29. Bartolozzi C, Cioni D, Donati F, Lencioni R. Focal liver lesions: MR imaging-pathologic correlation. Eur Radiol 2001;11(8):1374-1388.

30. Torbenson M. Review of the clinicopathological features of fibrolamellar carcinoma. Adv Anat Pathol 2007;14(3):217-223.

31. Fullerton GD, Potter JL, Dornbluth NC. NMR relaxation of protons in tissues and other macromolecular water solutions. Magn Reson Imaging 1982;1:209-226.

32. Reimer P, Tombach B. Upper abdomen: liver, pancreas, biliary system, and spleen. In Reimer P, Parizel PM, Stichnoth F-A eds: Clinical MR imaging. Berlin, Germany: Springer, 2006;272-318. 
33. Hussein SM. Melanoma liver metastases I: focal type. In: Liver MR imaging. Berlin, Germany: Springer, 2007;56-57.

34. Fujita M, Horinouchi T, Ishiguro S, et al. T2-shortening effect of fibrinogen inclusions on MR imaging of hepatocellular carcinoma: case report and experimental relaxation measurement. AJR Am J Roentgenol 2004;182:459-462.

35. Bartolozzi C, Lencioni R. Ethanol injection for the treatment of hepatic tumours. Eur Radiol 1996;6:682-696.

36. Sironi S, De Cobelli F, Livraghi T, et al. Small hepatocellular carcinoma treated with percutaneous ethanol injection: unenhanced and gadolinium-enhanced MR imaging followup. Radiology 1994;192:407-412.

37. Colagrande S, Politi LS, Messerini L, Mascalchi M, Villari N. Solitary necrotic nodule of the liver: imaging and correlation with pathologic features. Abdom Imaging 2003;28(1):41-44.

38. Stoupis C, Taylor HM, Paley MR, et al. The rocky liver: radiologic-pathologic correlation of calcified hepatic masses. Radiographics 1998;18:675-685.

39. Ichikawa T, Federle MP, Grazioli L, Madariaga J, Nalesnik M, Marsh W. Fibrolamellar hepatocellular carcinoma: imaging and pathologic findings in 31 recent cases. Radiology 1999;213:352-361.

40. Prasad SR, Wang H, Rosas H, et al. Fat-containing lesions of the liver: radiologic-pathologic correlation. Radiographics 2005;25(2):321-331.

41. Paulson EK, McClellan JS, Washington K, Spritzer CE, Meyers WC, Baker ME. Hepatic adenoma: MR characteristics and correlation with pathologic findings. AJR Am J Roentgenol 1994;163:113-116.

42. Stanley G, Jeffrey RB Jr, Feliz B. CT findings and histopathology of intratumoral steatosis in focal nodular hyperplasia: case report and review of the literature. J Comput Assist Tomogr 2002;26:815-817.

43. Eisenberg LB, Warshauer DM, Woosley JT, Cance WG, Bunzendahl H, Semelka RC. CT and MR imaging of hepatic focal nodular hyperplasia with peripheral steatosis. J Comput Assist Tomogr 1995;19:498-500.

44. Chaoui A, Mergo PJ, Lauwers GY. Unusual appearance of focal nodular hyperplasia with fatty change. AJR Am J Roentgenol 1998;171:1433-1434. 


\title{
The Hypointense Liver Lesion on T2-weighted MR Images and What It Means
}

\author{
Luís C urvo-Semedo, M D, et al
}

RadioGraphics 2009; e38 • Published online 10.1148/rg.e38 • Content Codes: GI MR

\section{Teaching Points}

- The T 2-weighted SI of methemoglobin depends on whether it is located in an intracellular or extracellular environment; the former results in low SI (marked T 2 shortening), while the latter causes high SI (less T 2 shortening). In later stages, hemorrhage may have low SI with both T 2- and T 1-weighted sequences since the intracellular forms of iron ferritin or hemosiderin provoke marked SI loss due to magnetic susceptibility effects.

- Intracellular iron particles cause local field inhomogeneities that accelerate the T $2 *$ of tissues by rapid dephasing of the transverse magnetization. This effect can be appreciated with sequences that are sensitive to magnetic field inhomogeneities, such as T 2-weighted spin echo or, particularly, T2*-weighted gradient echo.

- Free water molecules have unrestricted motion, whereas bound water (eg, associated with intracellular proteins) shows restricted motion. The hydrogen protons of bound water tumble with a frequency closer to the L armor frequency, causing more efficient $T 1$ and $T 2$ relaxation. This results in a shortening of both the T 1 and T 2 relaxation times of water.

- $\quad \mathrm{N}$ ecrotic areas may exhibit variable features on $M \mathrm{R}$ images, since liquefactive necrosis is highly hyperintense on T 2-weighted images, owing to increased water content, whereas coagulative (dehydrated) necrosis is characterized by low SI on T2-weighted images.

- Calcification typically has low SI with both T 1-weighted and T 2-weighted sequences; however, faint or tiny calcifications may not be detectable on $M \mathrm{R}$ images. 